ARTICLE

\title{
COMPOSITUM 1 contributes to the architectural simplification of barley inflorescence via meristem identity signals
}

Naser Poursarebani (iD ${ }^{1 凶}$, Corinna Trautewig ${ }^{1}$, Michael Melzer ${ }^{1}$, Thomas Nussbaumer 2,3 , Udda Lundqvist ${ }^{4}$, Twan Rutten ${ }^{1}$, Thomas Schmutzer (10) 1,5, Ronny Brandt', Axel Himmelbach', Lothar Altschmied (10 1, Ravi Koppolu1, Helmy M. Youssef ${ }^{1,5,6}$, Richard Sibout (10 7,8, Marion Dalmais ${ }^{9}$, Abdelhafid Bendahmane ${ }^{9}$, Nils Stein (i) ${ }^{1}$, Zhanguo Xin (1) ${ }^{10} \&$ Thorsten Schnurbusch (10) $1,5 \bowtie$

Grasses have varying inflorescence shapes; however, little is known about the genetic mechanisms specifying such shapes among tribes. Here, we identify the grass-specific TCP transcription factor COMPOSITUM 1 (COM1) expressing in inflorescence meristematic boundaries of different grasses. COM1 specifies branch-inhibition in barley (Triticeae) versus branch-formation in non-Triticeae grasses. Analyses of cell size, cell walls and transcripts reveal barley $\mathrm{COM} 1$ regulates cell growth, thereby affecting cell wall properties and signaling specifically in meristematic boundaries to establish identity of adjacent meristems. COM1 acts upstream of the boundary gene Liguleless 1 and confers meristem identity partially independent of the COM2 pathway. Furthermore, COM1 is subject to purifying natural selection, thereby contributing to specification of the spike inflorescence shape. This meristem identity pathway has conceptual implications for both inflorescence evolution and molecular breeding in Triticeae.

\footnotetext{
${ }^{1}$ Leibniz Institute of Plant Genetics and Crop Plant Research (IPK), Corrensstr, 3 OT Gatersleben, D-06466 Seeland, Germany. ${ }^{2}$ Technical University of Munich and Helmholtz Center Munich, Institute of Environmental Medicine, UNIKA-T, Neusäßer Str. 47, 86156 Augsburg, Germany. ${ }^{3}$ Helmholtz Zentrum München (HMGU), German Research Center for Environmental Health, Institute of Network Biology (INET), 85764 Neuherberg, Germany. ${ }^{4}$ Nordic Genetic Resource Center (NordGen), Smedjevägen 3, Box P.O. 41, SE-230 53 Alnarp, Sweden. ${ }^{5}$ Martin Luther University Halle-Wittenberg, Faculty of Natural Sciences III, Institute of Agricultural and Nutritional Sciences, 06120 Halle, Germany. ${ }^{6}$ Faculty of Agriculture, Cairo University, Giza, Egypt. ${ }^{7}$ Institut JeanPierre Bourgin, INRAE, AgroParisTech, Université Paris-Saclay, 78000 Versailles, France. ${ }^{8}$ INRAE, UR BIA, F-44316 Nantes, France. ${ }^{9}$ INRAE, CNRS, Institute of Plant Sciences Paris-Saclay IPS2, Univ Paris Sud, Univ Evry, Univ Paris-Diderot, Sorbonne Paris-Cite, Universite Paris-Saclay, 91405 Orsay, France.

10 USDA-ARS, Plant Stress and Germplasm Development Unit, Cropping Systems Research Laboratory, Lubbock, TX 79415, USA.

凶email: Poursarebani@gmail.com; Schnurbusch@ipk-gatersleben.de
} 
T he grass family (Poaceae), one of the largest angiosperm families, has evolved a striking diversity of inflorescence morphologies bearing complex structures, such as branches and specialized spikelets ${ }^{1}$. These structural features are key for sorting the grass family into tribes ${ }^{1}$. Current grass inflorescences are proposed to originate from a primitive ancestral shape exhibiting "a relatively small panicle-like branching system made up of primary and secondary paracladia (branches), each one standing single at the nodes" ${ }^{2}$ (Fig. 1a). This ancestral panicle-like inflorescence is also known as a compound spike $e^{3-5}$. Several independent or combined diversification processes throughout the evolutionary history of the grass family have resulted in the broad diversity of today's grass inflorescences ${ }^{2,3,6}$. Some tribes, e.g., Oryzeae (rice) and Andropogoneae (maize and sorghum), still display ancestral and complex compound shapes, keeping true-lateral long primary and secondary branches. Other grasses, such as Brachypodium distachyon, show lower inflorescence complexity with branch length and number reduced to lateral, small pedicels ending in only one multi-floretted spikelet (Fig. 1a-c). Inflorescences within the tribe Triticeae, e.g., barley (Hordeum vulgare L.), probably evolved from the ancestral compound spike into the typical unbranched spike (Fig. 1d). The spike displays the least-complex inflorescence shape due to the sessile nature of spikelets and reduction in rachis internodes ${ }^{2,7}$. Architectural variation is often manifested through subtle modifications of transcriptional programs during critical transitional windows of inflorescence meristem (IM) matura$\operatorname{tion}^{7,8}$ or functional divergence of key transcriptional regulators and/or other genes 9,10 . Identification of key genetic determinants is crucial for better understanding and explaining both the origin of grass inflorescence diversity and grass developmental gene evolution. Inflorescence developmental patterning controls pollination, grain set and grain number, and is thus highly relevant to agronomy as a target of natural and human selection.

A valuable toolkit to further explore such genetic determinants regulating inflorescence patterning in Triticeae is a collection of morphological barley mutants, induced by physical and chemical mutagens ${ }^{11}$. This collection includes compositum (com) barley mutants displaying branched spikes and their corresponding near-isogenic lines (NILs) ${ }^{12}$. Eight of such NILs have been reported so far ${ }^{12}$; however, only one, NIL com 2 , has been characterized. The underlying gene of com 2 encodes an AP2/ERF transcription factor orthologous to maize BRANCHED SILKLESS 1 (BD1) with a conserved function of branch suppression across grasses ${ }^{13}$.

In this study, we have conducted a detailed phenotypic inspection of the barley com 1 NIL mutant also displaying noncanonical, i.e., branched spike morphology. We identify and characterize the underlying boundary forming protein, a grassspecific TCP transcription factor, and present evidence that COM1 in barley has evolved a function opposite to its known orthologous proteins in maize and rice, ZmBAD1/WAB1 and OsREP1/DBOP, respectively ${ }^{14-17}$. Mororeover, we find that its orthologous protein in sorghum also functions oppositely to barley, while its function in Brachypodium distachyon appears less diversified. Unlike in these non-Triticeae grasses, in which branch-formation is commonly promoted, barley COM1 inhibits spike-branching most likely by affecting meristematic signaling via changing cell wall properties of meristematic boundary cells. The generated double mutant (DM) of com1.a/com 2.g provides evidence that DM plants outperformed both single mutants and wild type plants in supernumerary spikelet formation, and as a consequence, in grain number per spike. Thus, our findings may spur further interests for grass inflorescence evolution but similarly for improving grain number.

\section{Results}

Atypical for Triticeae-barley com1.a forms a branched inflorescence. Barley (and other Triticeae) wild-type (Wt) spikes are typically unbranched and composed of sessile, single-flowered spikelets arranged in a regular distichous fashion of two opposite rows directly attached to the main inflorescence axis, i.e., rachis (Fig. 1e). In a mature barley spike, three spikelets per rachis node are visible. Each spikelet initiates from a single meristematic mound first detectable at the triple mound (TM) stage during early reproductive development (Fig. 1f). Thus, the TM corresponds to three spikelets meristems (SMs): one central (CSM) and two lateral (LSM) SMs. The differentiating primordia are followed by several consecutive meristematic and developmental stages, e.g., glume primordium (GP; Fig. 1g), lemma primordium (LP; Fig. 1h), and stamen primordium (SP; Fig. 1i, j) ${ }^{18}$.

To provide deeper insights into the genetic basis defining inflorescence architecture in barley (Triticeae), we conducted a detailed phenotypic inspection of a NIL of com barley (com1.a) mutant displaying a branched spike (Fig. 1k). The original com 1.a spike-branching mutant was first discovered after simultaneous mutagenesis using EMS and neutron radiation of $c v$. Foma. It was later backcrossed $\left(\mathrm{BC}_{6}\right)$ to a two-rowed barley $c v$. Bowman $(B W)^{12}$ to create the aforementioned NIL, BW-NIL(com1.a) (Supplementary Fig. 1A, B). Thus, we hereafter refer to the BWNIL(com1.a), as com1.a mutant. The inflorescence in this com1.a mutant resembles an ancestral compound spike (Fig. 1k) but lacks an organ called pulvinus (Fig. 1n-o). In non-Triticeae grass species, the pulvinus is present at the axil of lateral long branches in panicles and compound spikes, defining branch angle extent (Fig. 1a-c, in brown). We observed differences in spike shape between BW and com1.a during early spike differentiation at the late triple mound (TM) to early glume primordium (GP) stage; the mutant CSM is elongated (Fig. 1p vs. Wt in g), becoming more apparent during later reproductive stages of late glume primordium (Fig. 1q) onwards (Fig. 1r, s). At LP, predominantly in the basal part of the spike, meristems of the central spikelet positions undergo evidently SM identity loss, displaying branchor IM-like meristems (Fig. 1r). Instead of generating florets, the meristem continues to elongate and rather functions as an indeterminate spikelet multimer in the form of a primary branchlike structure (Fig. 1t). Such branch-like structures occasionally replace other spikelet-related organs, such as the rachilla primordium (RP, the spikelet axis, in Fig. 1s; the possible origin for extended rachilla visible at maturity; Fig. 1l, m) or glumes (Fig. $1 \mathrm{t}$ in purple). The com 1 branching phenotype resembles that of the previously described $c o m 2^{13}$, in which the formation of branch-like structures results from lack of SM identity (see belowCOM1 inhibits inflorescence branching partially independent to COM2).

COM1 restricts palea cell size by thickening their cell walls. In barley, grains are enclosed by two bract-like organs, i.e., lemma and palea, that are part of the floret and provide protection to the developing grain. (Fig. $1 \mathrm{~m}$ ). Besides the branching phenotype, com1.a has a deviation in adaxial palea morphology by exhibiting a flat plane (Fig. 1u) versus the conventional distinct infolding observed in paleae of BW (Fig. 1v), cv. Foma, and wild barley (H. vulgare subsp. spontaneum). This deviation was visible in all paleae independent of their position along the spike. Histological analyses using cross sections of paleae middle-areas (Fig. 1u) revealed distinct features of com1.a in which sclerenchymtous cells, in particular, appeared to be expanded in size and most likely also in numbers (Fig. 1w-x); however, we did not determine cell numbers quantitatively. Cell expansion is thought to be tightly linked to cell wall extensibility ${ }^{19,20}$. We used transmission 

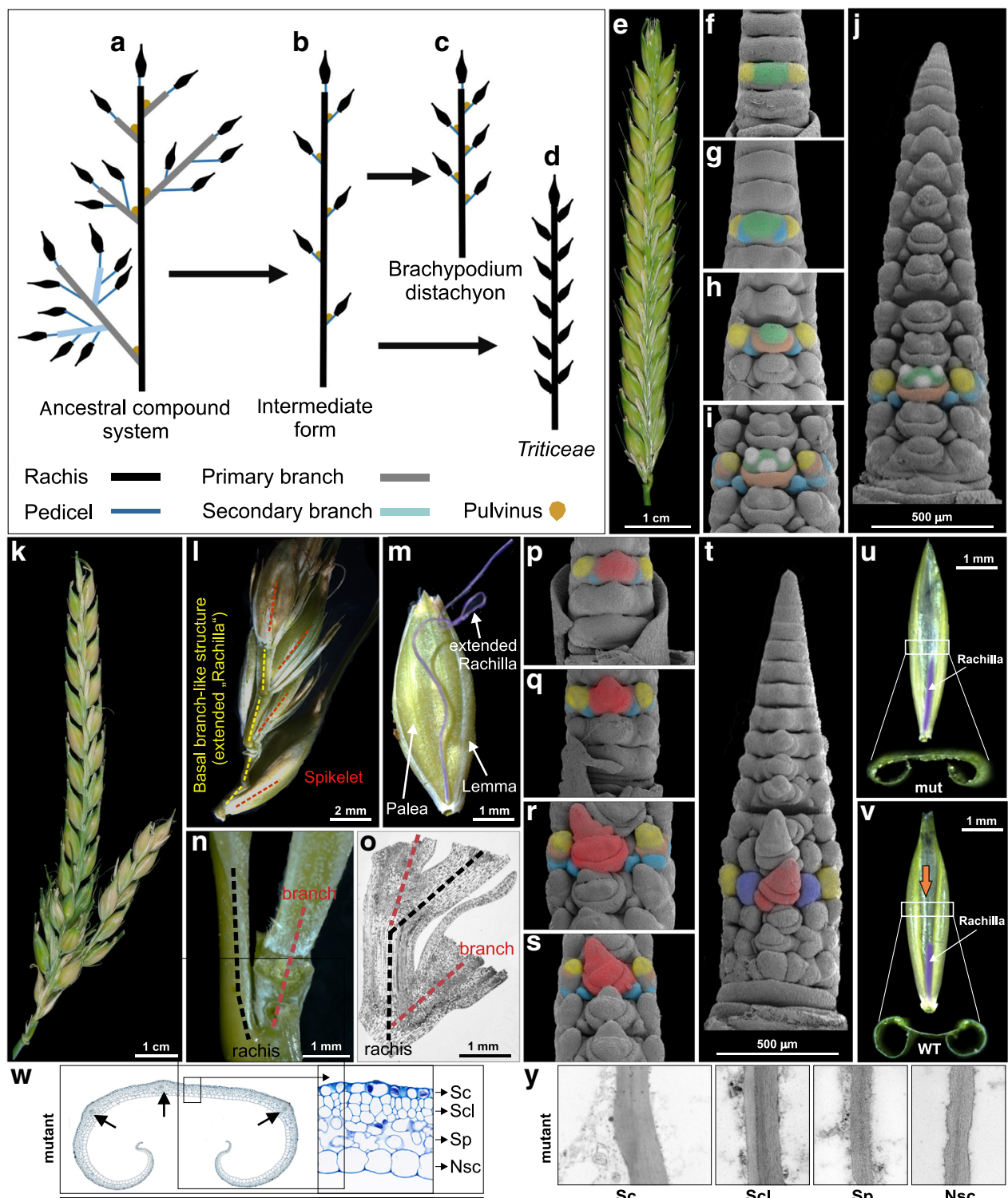

$\mathbf{X}$

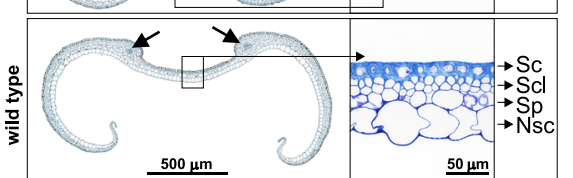

$\mathbf{Z}$

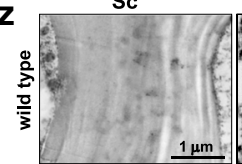

$$
\begin{array}{ll}
\square \text { Glume Primordium; GP } & \square \text { Central Spikelet Meristem; CSM } \\
\square \text { Lemma Primordium; LP } & \square \text { Lateral Spikelet Meristem; LSM } \\
\square \text { Stamen Primordium; SP } & \square \text { IM-like branch } \\
\square \text { Uncommonly enlarged meristem } & \Rightarrow \text { infolding area } \\
\text { in place of Glume Primordium } &
\end{array}
$$

Fig. 1 Proposed evolutionary pattern of grass inflorescences, and barley spike/palea morphology in wild-type and com1.a mutant. a-d Model for grass inflorescence evolution from ancestral compound form to spike in Triticeae; reprinted from Vegetti and Anton ${ }^{2}$ with kind permission from Elsevier. e Spike morphology of wild-type ( $\mathrm{Wt}$ ), two-rowed barley $\mathrm{cv}$. Bowman. $\mathbf{f - i}$ SEM imaging of the early developmental stages in immature Wt spike; triple mound: TM (f) glume primordium: GP ( $\mathbf{g}$ ) lemma primordium: LP (h) and stamen primordium: SP (i). Images are taken from basal nodes where a single node is used for color coding. $\mathbf{j}$ Dorsal view of whole immature Wt spike at stamen primordium stage. $\mathbf{k}$ Branched spike of BW-NIL(com1.a) mutant at maturity. $\mathbf{I}, \mathbf{m}$ Depicted is a small, spike-like branch structure, arisen from the central spikelet position due to loss of CSM identity, from intense (I) to weak appearance as an extended (ext.) rachilla $(\mathbf{m}), \mathbf{M}$ also depicts a developing grain enclosed by lemma and palea. $\mathbf{n}, \mathbf{o}$ Lack of pulvinus at the base of a branch in BW-NIL(com1.a) mutant spike (n) supported by histological imaging (o). p-s Developmental stages of immature BW-NIL(com1.a) mutant spike from early GP (p), GP (q), to LP (r) and early SP (s) taken from the basal nodes. t Dorsal view of whole immature BW-NIL(com1.a) mutant spike at early stamen primordia. u Longitudinal adaxial view of the palea in BW-NIL(com1.a); white rectangle corresponds to the area used to take sections for histological analysis and to the lower image depicting the flat-plane surface of a palea cross section. mut stands for mutant (v) Longitudinal adaxial view of the palea in $\mathrm{Wt}$; the lower image corresponds to the infolding surface of a palea cross section. $\mathbf{w}, \mathbf{x}$ Histological analyses of transverse sections (from $\mathbf{u}, \mathbf{v}$; white rectangles) of the palea in BW-NIL(com1.a) (w) and Wt (x). Paleae are from spikelets shortly before anthesis. $\mathbf{y}, \mathbf{z}$ TEM based imaging of walls of paleae cells in BW-NIL(com1.a) (y) versus Wt (z). 


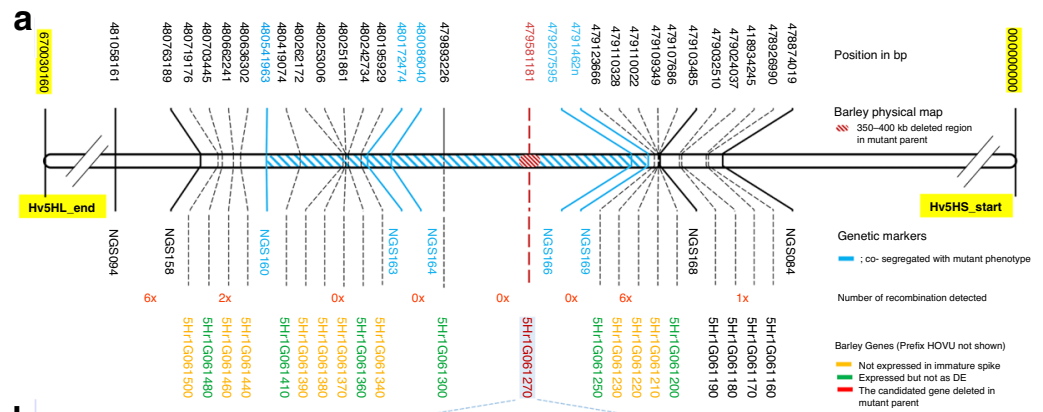

b
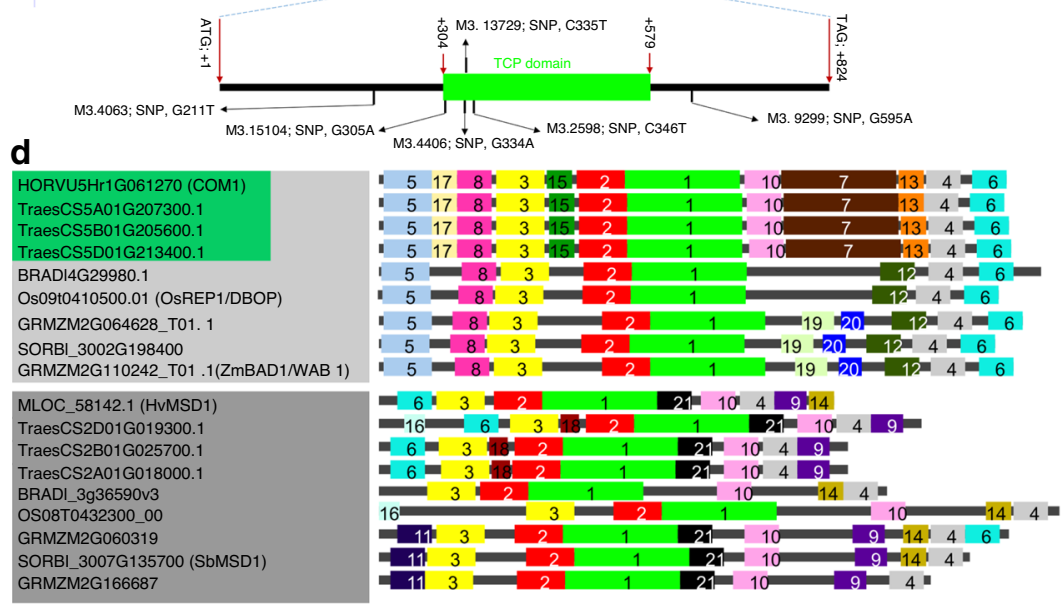

C

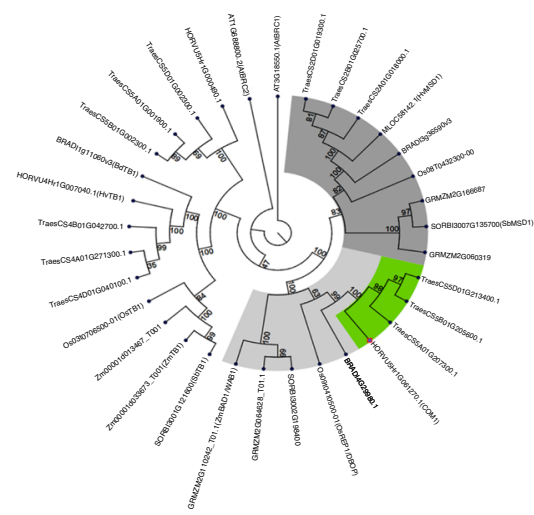

aа

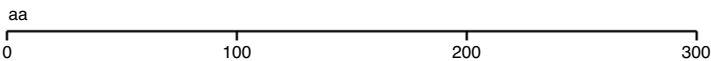

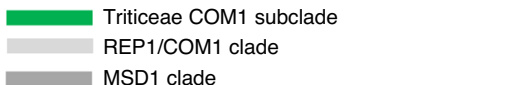

Species IDs are abbreviated as gene 10 prefixes ; HORVU; Hordeum vulgare, Traes; Triticum aestivum, BRADI; Brachypodium distachyon, Os; Oryza sativa, SORBI; Sorghum bicolor, GRMZM (ZM); Zea mays, At; Arabidopsis thaliana

Fig. 2 Map-based cloning, phylogenetic analysis, and protein structural variation of COM1. a Physical and genetic maps of com1.a from 100 recombinant plants or 6,000 gametes. A single gene (red; HORVU5Hr1G061270, a single-exon TCP transcription factor) was the strongest candidate and deleted in the mutant parent com1.a. b COM1 gene model containing one TCP DNA binding domain (green box). Six barley TILLING alleles are shown with prefix M3. c UPGMA phylogenetic tree, using 1,000 bootstrap replications, of COM1 homologs (highlighted in light gray) and paralogs (in dark gray) appeared as first- and second-best hits, respectively, in the blast search. Bootstrap values (in percentage) are shown within the circular cladogram along the edges of the branches. d Evolutionarily conserved motifs, among COM1 homologous and paralogous proteins (presented as phylogenetic tree in c) using the tool SALAD. Each colored box represents a different and numbered protein motif. For example, motif 1 in light green represents the TCP domain. Motifs 7, 13, 15, and 17 of the REP1/COM1 clade are specific to the Triticeae. (see also Supplementary Fig. 6). .

electron microscopy (TEM) to verify whether com1.a palea cells had altered cell wall features. Notably, mutant palea cells had clearly thinner cell wall structures, thus fewer mechanical obstructions for cell expansion, implicating that COM1 functions as a regulator of cell growth via cell wall modifications (Fig. 1y, z and Supplementary Fig. 2). Moreover, mutant paleae generally formed three vascular bundles (VB) (Fig. 1w) compared with two VBs in BW (Fig. 1x). By analogy to changes in palea cell walls, such alterations might also explain the rescission of SM identity provided that COM1 similarly affects cell wall integrity in meristematic cells, e.g., SM or boundary cells (cells separating inflorescence meristem, IM, from SMs) (see below-barley COM1 function evolved to affect boundary signaling as well as the transcriptome analysis).

COM1 encodes a class II, subclass CYC/TB1 TCP transcription factor. To investigate the genetic basis of the com1.a phenotype, we constructed a genetic linkage map by screening $\sim 6,000$ gametes for recombination events in an $\mathrm{F}_{2}$ population (Bowman $\times$ com 1.a) followed by further analysis of $\mathrm{F}_{3}$ families. Fifteen critical recombinant $\mathrm{F}_{2}$-derived $\mathrm{F}_{3}$ families (i.e., 16 plants per family) were further analyzed (Supplementary Data 1 and 2; Supplementary Tables 1; Supplementary Fig. 1C-F), delimiting a $\sim 1.4 \mathrm{Mb}$ interval carrying eight genes; a predicted transcription factor (HORVU 5Hr1G061270) is entirely absent in coml.a, likely due to an induced deletion (Fig. 2a). The remaining seven genes either were not expressed or not differentially regulated between Wt and com1.a mutant (see below-the transcriptome analysis).

To validate our candidate gene (HORVU 5Hr1G061270), we sequenced it in a set of 20 induced spike-branching mutants and in a barley TILLING population of two-rowed barley, cv. Barke. Resequencing of branching mutants, using both CDS and promoter specific primer pairs (Supplementary Data 1), revealed that five of them, i.e., Mut.3906, int-h.42, int-h.43 and int-h.44, and com1.j, lost the same transcription factor as was found missing in the com1.a mutant (Fig. 3 and Supplementary Data 3). All five mutants also showed the flat-palea phenotype, previously observed in the com1.a mutant (Fig. 3). Allelism tests of com1.a with Mut.3906 indicated that they are allelic to each other. Furthermore, PCR-screening of the TILLING populations for the CDS of the candidate gene revealed four homozygous M3 plants (M3.15104, M3.4406, M3.13729, and M3. 2598) carrying SNP mutations inside the DNA binding domain (Fig. 2b). Additionally, two heterozygous M3 lines M3.4063 and M3.9299 with SNP mutation outside the domain were also identified (Fig. 2b). All six SNP mutations caused amino acid substitution in conserved positions (Fig. 2b). They all transmitted a branched spike revealed by the phenotypes of the corresponding M4 and M5 homozygous plants (Fig. 4 and Supplementary Figs. 3 and 4; Supplementary 

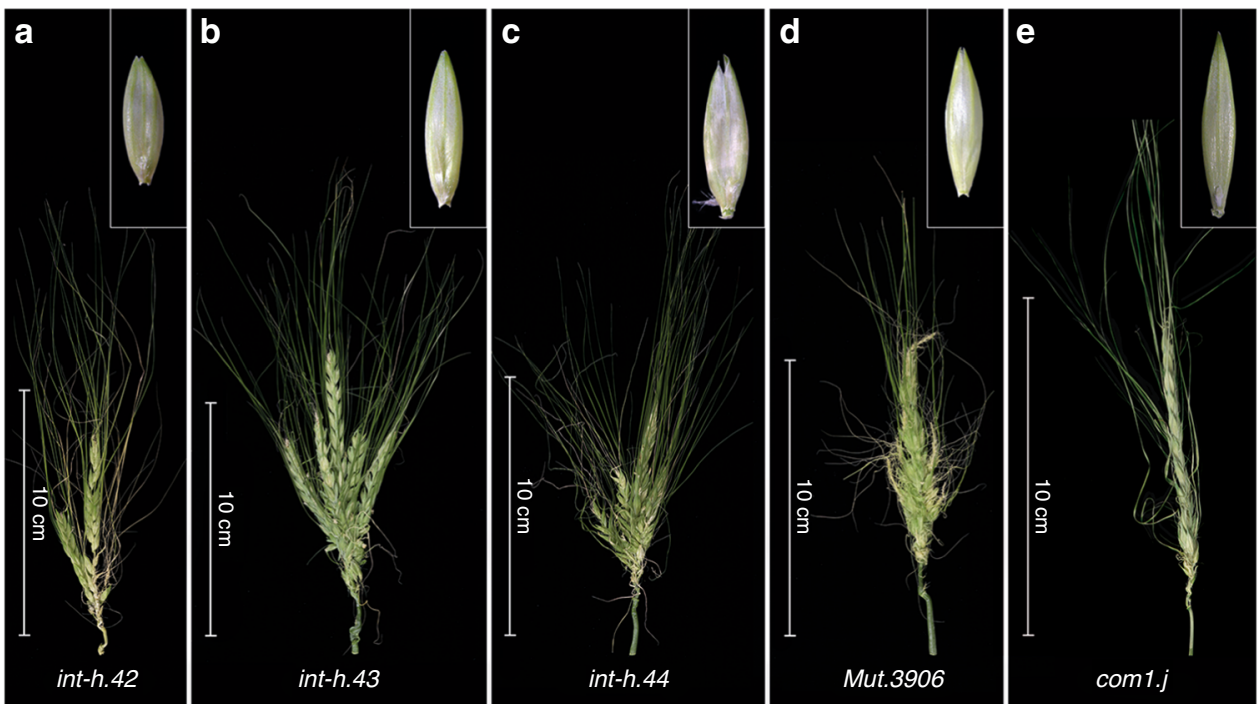

Fig. 3 Barley COM1 mutant alleles showing spike-branching and paleae phenotypes. a-e Different induced mutant alleles identified by resequencing of CDS and putative promotor region of COM1. The corresponding palea is shown in the upper-right side of each spike image. See also Supplementary Data 1 and 3.
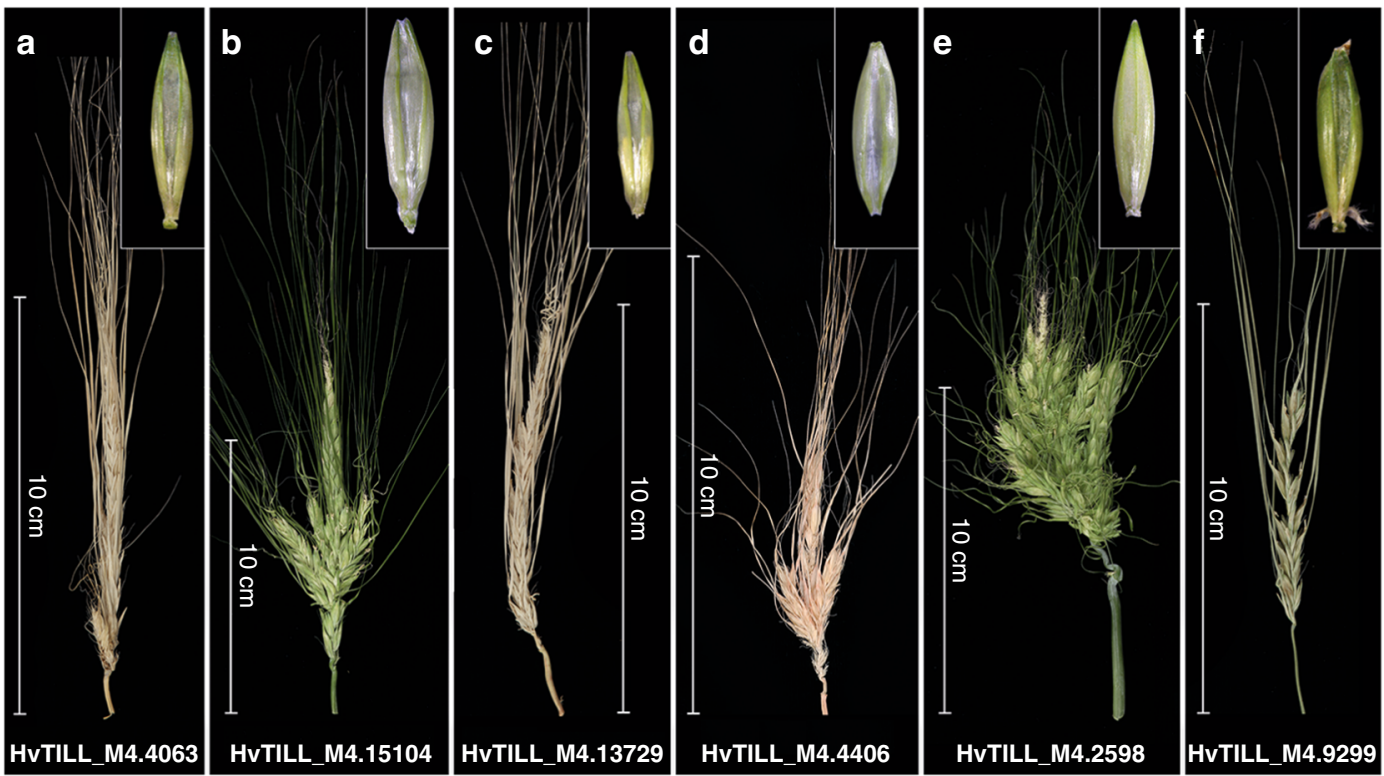

Fig. 4 Spike and paleae phenotypes of barley TILLING mutants. a-f A Representative display of branch formation of the six barley TILLING mutants derived from barley $\mathrm{cv}$. Barke. The corresponding palea is shown in the upper-right side of each spike image. HvTILL stands for Hordeum vulgare TILLING line. For the underling protein sequence lesion, please refer to Supplementary Fig. 3.

Data 3). Interestingly, from the six TILLING mutants only two, with mutation within the TCP domain, showed either a flat-palea phenotype with a complete loss of the infolding (line 2598, exhibiting also the most severe branching) or only a mild change in the palea shape (line 4406) (Figs. 2b, 4). Thus, penetrance of the flat-palea mutant phenotype depended on the type and position of the amino acid substitution (Supplementary Fig. 3, legend). Together, these data confirmed unambiguously that the transcriptional regulator was responsible for the spike-branching and palea phenotypes in com1.a. Annotation analysis of the COM1 protein showed that it belongs to the plant-specific TCP (Teosinte branched 1 (TB1)/Cycloidea/Proliferating Cell Factor) transcription factor family; COM1 contains 273 amino acids and features one basic helix-loop-helix TCP domain (Fig. 2b). Proteins of the TCP family fall into two classes, with COM1 belonging to class II, subclass CYC/TB1 $1^{21,22}$.
Barley COM1 function evolved to affect boundary signaling. We next asked whether COM1 has experienced functional conservation or divergence within the grasses and whether its sequence composition supports possible functional alteration. We used the comprehensive phylogenetic analyses available for grass $\mathrm{TCPs}^{22,23}$ (and the references therein) as a starting point for our own COM1-specific phylogenetic analyses. We searched for homologs and paralogs of COM1 in sequenced grass genomes, including rice, maize, sorghum, hexaploid wheat and Brachypodium distachyon, as well as Arabidopsis thaliana (Fig. 2c). The homolog of maize TB1, obtained from the aforementioned grasses, was added as an out-group to the phylogeny. Our sequence searches and the phylogenetic analysis confirmed that COM1 is restricted to grasses (Fig. 2c) as reported previously $14,15,24$. The homologs of COM1 in maize and rice were reported previously as ZmBAD1/WAB1 and 
Table 1 Functional variation of COM1 homologs observed among grass species.

\begin{tabular}{|c|c|c|c|c|c|c|}
\hline Species (Name) & $\begin{array}{l}\text { Gene function in } \\
\text { boundary }\end{array}$ & $\begin{array}{l}\text { GEa on branch } \\
\text { formation }\end{array}$ & $\begin{array}{l}\text { GE on pulvinus } \\
\text { size/formation }\end{array}$ & $\begin{array}{l}\text { GE on growth of } \\
\text { palea cells }\end{array}$ & $\begin{array}{l}\text { GE on number of } \\
\text { VB }^{\mathbf{b}} \text { in palea }\end{array}$ & $\begin{array}{l}\text { GE on Pollen } \\
\text { fertilityc }\end{array}$ \\
\hline Barley (COM1) & signaling & Inhibition & Absent $^{d}$ & Inhibitione & Promotion & Normal \\
\hline $\begin{array}{l}\text { Brachypodium (BdWAB1/ } \\
\text { BAD1) }\end{array}$ & formation ${ }^{f}$ & No effect & Promotion & No effectg & Promotion & Normal \\
\hline Rice (REP1) & formation & Not reported ${ }^{\mathrm{h}}$ & Promotion & Promotion & Promotion & Reduced \\
\hline $\begin{array}{l}\text { Sorghum (SbWAB1/ } \\
\text { BAD1) }\end{array}$ & formation & Promotion & Promotion & No effect & Promotion & Reduced \\
\hline Maize (WAB1/BAD1) & formation & Promotion & Promotion & Not reported & Not reported & Not reported \\
\hline \multicolumn{7}{|c|}{$\begin{array}{l}\text { aGE stands for functional gene effect. } \\
\text { bVB stands for vasculature bundles. } \\
\text { cRevealed by grain setting measurements as a proxy. } \\
\text { dPulvinus is typically absent in Wt. spike of Triticeae including barley as well as in the branched mutant spikes. } \\
\text { eApparent at the longitudinally middle palea part resulting in the formation of the infolding. } \\
\text { fRefers to the formation of a boundary between pulvinus and the lateral branch without which fusion of the two happened; reflects intermediate evolutionary phylogenetic position of Brachypodium } \\
\text { among grasses. } \\
\text { gNot visible at the microscopic level. } \\
\text { hPerhaps because the rice cultivars used in the corresponding studies (cv. Nipponbare and cv. 9522) are known to exhibit panicles with acute lateral branches. }\end{array}$} \\
\hline
\end{tabular}

OsREP1/DBOP (60.3\% and $65.5 \%$ sequence similarity to COM1), respectively ${ }^{14-17}$. Except for maize, none of the COM1 homologs showed a duplication after speciation (e.g., no in-paralogs resulting from within-genome duplication, ${ }^{25}$ ). Furthermore, COM1 seems to be a paralog (e.g., out-paralog 25 that refers to duplication before speciation; see Supplementary Fig. 5A, B) of the sorghum gene SbMSD1 (44.1\% sequence similarity to COM 1$)^{26}$. Functional characterization of COM1 homologs is only available for maize and rice (Table 1$)^{14,15,17}$.

Maize $B A D 1 / W A B 1$ transcripts are mainly detected at the IMto-BM (branch meristem) boundary region as well as between pulvinus and lateral branches (in Fig. $3 \mathrm{j}$ of Bai et al. ${ }^{15}$ ). Consequently, loss-of-function $b a d 1 / w a b 1$ mutants display organ fusion (a known boundary formation defect) resulting in reduced branch number (from 5.8 in Wt to 1.3 in mutant siblings) and angle size, and more upright tassel branches ${ }^{14,15}$. This gene was dubbed a boundary formation gene promoting lateral meristem (e.g., branch) and axillary organ (e.g., pulvinus) formation in $\mathrm{Wt}$ maize $^{14,15}$.

Our phylogenetic analysis identified orthologs of COM1, in both sorghum and Brachypodium distachyon (Fig. 2c). To further expand our knowledge about COM1 function within nonTriticeae grasses, we used a TILLING approach. We first screened a TILLING population in sorghum originating from $c v$. BTx623. The sorghum Wt inflorescence, a panicle, consists of a main rachis on which many primary, secondary as well as sometimes tertiary branches develop (Fig. 5a). Similar to maize, sorghum plants possess a pulvinus to regulate branch angle. The TILLING analysis revealed one mutant (ARS180 line; A144T) with both upright panicle branches $\left(10.95^{\circ}\right.$ in Wt vs. $5.2^{\circ}$ in mutant, $P \leq$ 0.001 ; Fig. 5a-g and Supplementary Table 2) and reduced primary branch number per node, i.e., whorls of branches (5.1 in Wt vs. 4.3 in mutant, $P \leq 0.05$; Supplementary Table 3). Measurement of branch angle was used as a proxy for pulvinus development (Supplementary Table 2). These data suggest a similar positive role of sorghum BAD1/WAB1 in pulvinus development and branch initiation/formation, revealing functional conservation of the protein between sorghum and maize. Moreover, we detected no obvious change in sorghum palea morphology except one additional vascular bundle, similar to maize and barley (Table 1).

The rice homolog of COM1, OsREP1/DBOP, shows a major effect in promoting palea identity, growth and development, with no effect on branch angle or branch initiation ${ }^{16,17}$. Loss-offunction mutants display smaller paleae due to less differentiation and severely reduced size of palea cells; a clear contrast to palea defects in barley (Table 1). Our TILLING analysis of COM1 homologs in Brachypodium distachyon (for its Wt inflorescence shape see schematic Fig. $1 \mathrm{c}$ and $6 \mathrm{~d}$ ) identified several mutants. Phenotypic investigation of two mutants (5446: Q116* and 8373: S146N) (Supplementary Data 3 and Supplementary Note 1) revealed similar phenotypes to the aforementioned non-Triticeae species (Table 1) (Fig. 6a-i). Similarly, we observed a palea defect (Fig. 6a, b); but histological analyses revealed no changes in cell expansion, except the formation of one additional vascular bundle in each mutant (Fig. 6g, h). We also observed a reduction in branch angle because of smaller or absent pulvini (Fig. 6c-f); however, the number of lateral branches was not altered in either Brachypodium mutants (Fig. 6j-1). In conclusion, COM1 homologs within non-Triticeae grasses primarily promote boundary formation and cell differentiation (as in rice palea)/ proliferation (as seen for pulvinus) (Table 1); but similarly promote the formation of lateral axillary organs, e.g. branch or pulvinus, that contribute in maintaining a complex inflorescence architecture.

To better understand the contrasting COM1 function of branch-inhibition in barley versus branch-formation in nonTriticeae grasses, we analyzed barley COM1 expression using qRT-PCR and semi-qPCR (Fig. $7 \mathrm{a}-\mathrm{c}$ ) followed by mRNA in situ hybridization (Fig. $7 \mathrm{~d}-\mathrm{g}$ ). Barley COM1 transcripts were detected in paleae (Fig. 7c, f, g), VB of the rachis (Fig. 7e), and importantly at the base of forming SMs throughout the boundary region separating SMs from IM (IM-to-SM boundary) and between lateral and central SMs (Fig. 7e, f), similar to non-Triticeae grass species, e.g., maize. This expression pattern suggests the involvement of barley COM1 in specification of the spikelet meristematic boundary. However, since central and lateral spikelets do not fuse into each other or to the IM in the barley com1 mutant (as long branches do fuse to the IM in maize or sorghum mutants i.e., lowering the branch number per mutant IM), barley COM1 may not be involved in boundary formation per se but perhaps rather in boundary signaling (see belowtranscriptional results and discussion) ${ }^{27}$. Recently acquired protein motifs specific to Triticeae COM1 may support this functional difference (Fig. 2d motifs 7, 13, 15 and 17; Supplementary Fig. 6).

We then checked whether natural selection has acted upon barley COM1 sequence composition and function, and consequently the formation of unbranched spikes in barley. Resequencing of the barley COM1 coding sequence in a panel of 146 diverse barley landraces and 90 wild barleys ${ }^{28,29}$ revealed very little natural sequence variation (site diversity of $\mathrm{pi}=0.0006$ ). 
a

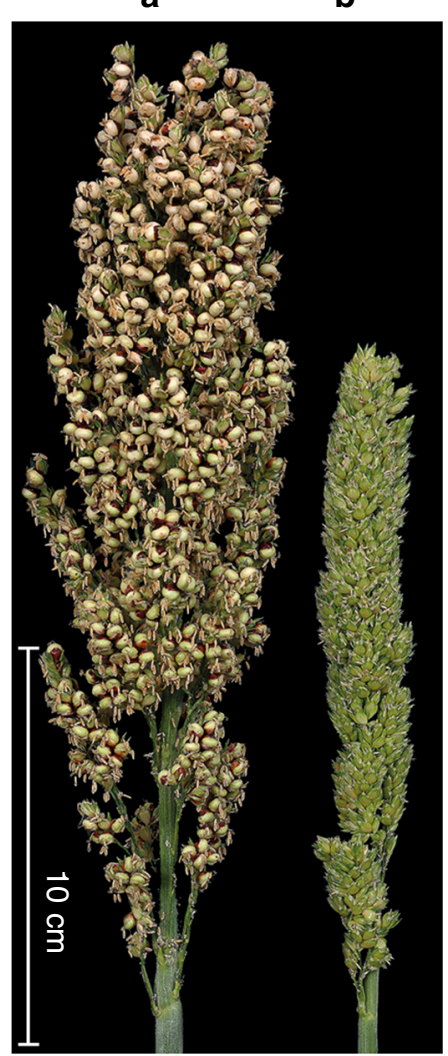

C

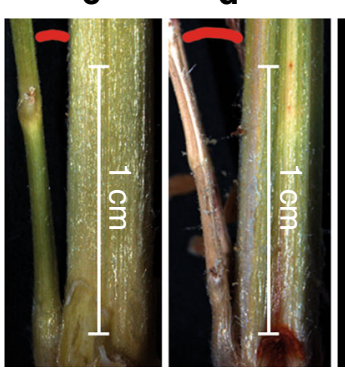

e

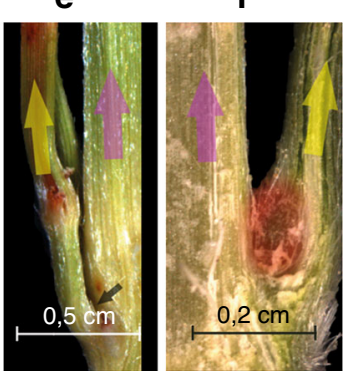

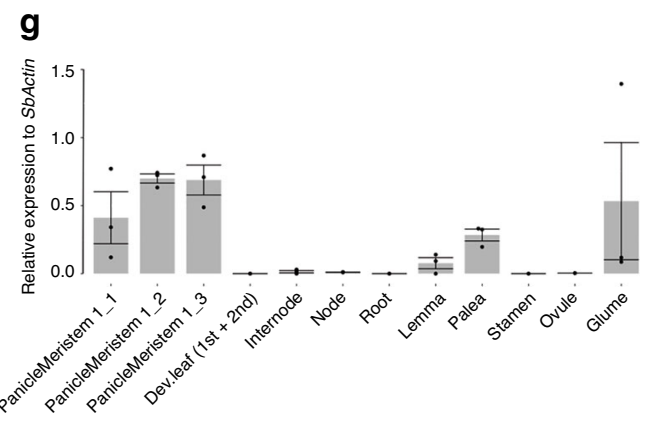

Fig. 5 Inflorescence morphology and the gene expression pattern in sorghum. a Sorghum inflorescence shape in Wt cv. BT623. b Compact sorghum inflorescence in TILLING mutant ARS180 showing severe reduction in grain setting (Supplementary Table 2; also reported for rep1 mutant in rice, see Table 1). c Acute branch angle in mutant ARS180 versus d expanded branch angle of Wt ( $5.2^{\circ}$ in mutant vs. $10.95^{\circ}$ in $W t, P \leq 0.001 ;$ see Supplementary Table 2). due to the lack or small size of the pulvinus. e Depicts lack of pulvinus at the base (black arrow) of the mutant lateral branch versus its presence (red, roundish area) in Wt (f). Arrows in yellow and pink represent the lateral primary branch and rachis, respectively, in both (e, f). $\mathbf{g}$ RT-qPCR of SbBAD1/ WAB1 in organs of Wt plants. 1_1, 1_2 and 1_3 represent first, second, and third branch meristem stages, respectively. Data collected from $n=3$ biologically independent experiments, $n=15-25$ plants per experiment, mean \pm SE of three biological replicates.

Eleven SNPs resulted in a simple 12-haplotype network (Supplementary Fig. 7) comprising only two main haplotypes, neither of the 12 showed mutant spike or palea phenotypes (Supplementary Fig. 7). This suggests that barley COM1 underwent purifying natural selection. We, therefore, expect that selection may have contributed to maintaining barley's slimmeddown inflorescence shape.

COM1 inhibits inflorescence branching partially independent to COM2. We had previously reported a branch suppressor protein, the AP2/ERF transcription factor COM2, with a conserved function across grass species ${ }^{13}$. COM2 expresses in an arclike region between central (CS) and lateral spikelets (LS) as well as between the $\mathrm{SM}$ and the emerging $\mathrm{GP}^{13}$. As the com 1 phenotype resembles that of com 2 (Fig. 8a-c), we developed and characterized BW-NIL(com1./com2.g) double mutants (e.g., DM, or com1./com 2.g double mutant) to study their interactions in regulating branch inhibition in barley.

We first performed a comparative SEM-based image analysis in immature spikes of the two single and the DM mutants. As illustrated above, SEM of the two single mutants revealed similar branched phenotypes by generating a simple branching structure (SBS) in which the CSMs located at the more basal nodes lost identity and converted into inflorescence-like branches (for a typical SBS see; Fig. 8b, c). SBS also included homeotic transformations of glumes (Fig. 8b, c, in purple). In contrast, DM immature spikes revealed interesting observations, including loss of identity and conversion of LSMs into branch-like structures, in addition to the loss of identity in CSMs (Fig. 8d). These conversions were observed in all nodes; not only in basal ones. Furthermore, glume primordia that only underwent an occasional homeotic transformation in basal nodes of single mutants (in purple, Fig. 8b, c) were also converted into IM-like meristems at all nodes of a DM spike (in purple, Fig. 8d). Therefore, a mixed meristematic re-organization per immature DM spike was observed representing a tentative ten-rowed barley spike (Fig. 8d and the legend); and thus, a rather complex branching structure (CBS) throughout the entire spike.

To further characterize the three genotypic classes, i.e., DMs, com1.a and com2.g, we also compared spike morphology at maturity. A set of 20 plants per class as well as 20 wild type $c v$. Bowman plants were grown to perform comparative phenotyping of mature spikes. Our visual inspection of the two single mutants at maturity revealed three types of spike forms, including $\mathrm{Wt}$ (Fig. 8e), SBS (Fig. 8f, g; typical SBS branching forms) and CBS (Fig. $8 \mathrm{~h}$ ). In case of Wt inflorescence architecture, only $3.7 \%$ of the com1.a spikes per family displayed this class, while com $2 . g$ showed a higher frequency of $22 \%$. As expected, the SBS was the most frequent phenotypic class in both single mutant families with 91 and $73 \%$ of the spikes per family in com1.a and com $2 . g$, respectively. Interestingly, both single mutants also showed a low level of CBS with similar frequencies (com1.a, 5.3\%; com2.g, 5.1\%) that were mostly visible in small late tillers. Thus, com1. a mutant plants showed a higher phenotypic penetrance for 

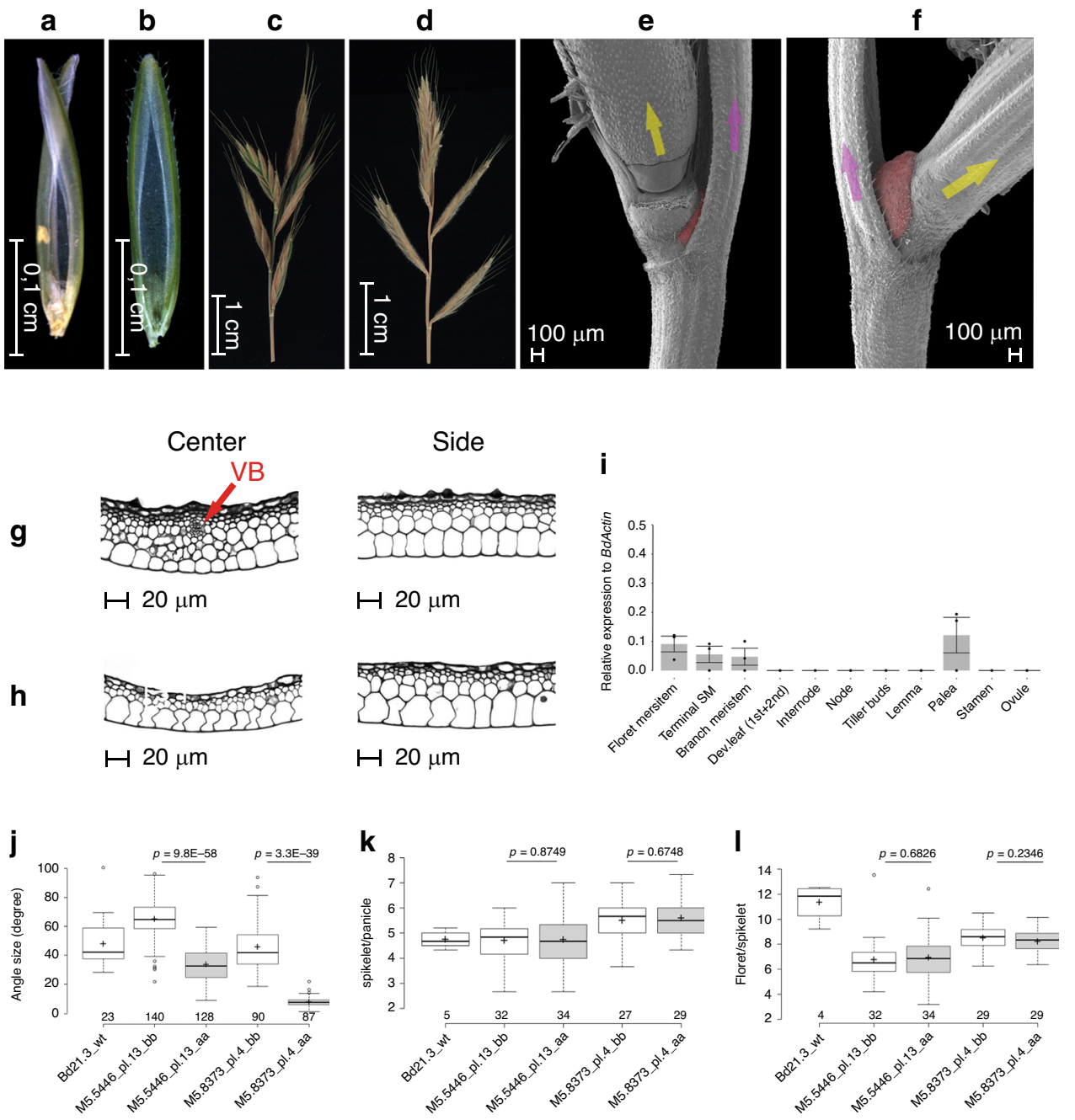

Fig. 6 Inflorescence morphology and the gene expression pattern in Brachypodium. a Paleae of Brachypodium mutants show scissor-like structures that collapse easily due to external mechanical pressure; b normal/solid palea structure in Wt plants. c Brachypodium mutant inflorescence with compact shape due to acute branch (spikelet) angles; $\mathbf{d}$ Brachypodium Wt inflorescence with normal architecture of expanded branch angle as result of normal growth with pulvinus. e SEM view of an abnormal tiny pulvinus (in red) of a Brachypodium mutant versus an intact normal-sized pulvinus (in red) in Wt (f); arrows in yellow and pink represent the lateral branch and rachis, respectively, both in $(\mathbf{e}, \mathbf{f})$. $\mathbf{g}$ Histological view of transverse section of Brachypodium mutant palea as compared to $\mathrm{Wt}$ (h); Brachypodium mutant has an extra VB in the center part (red arrow) that is lacking in Wt. Center refers to the collapsed middle part, while Side refers to the flanking intact area (the blades of the scissor; see part $\mathbf{a}$ ). $\mathbf{i}$ RT-qPCR of BdBAD1/WAB1 gene expression across meristematic stages and organs in Wt. Data collected from $n=3$ biologically independent experiments, $n=15-25$ plants per experiment, mean \pm SE of three biological replicates. $\mathbf{j}$ Branch angle measurement in Brachypodium as proxy for pulvinus size. $\mathbf{k}$ Number of spikelets per individual Brachypodium inflorescence (panicle). I Number of florets per spikelet in Brachypodium. In $\mathbf{j}-\mathbf{l}$; data are from contrasting M6 homozygous TILLING lines of Brachypodium; $\mathrm{aa}$ and bb refer to homozygous mutant (aa) and Wt (bb) alleles from the same family (Supplementary Note 1). Per box plot, plus signs show the means while center bold-lines show the medians; box limits indicate the 25th and 75th percentiles; whiskers extend 1.5 times the interquartile range, outliers are represented by circles. Values above $x$-axis indicate number of angles measured in $(\mathbf{j})$, and number of plants in $(\mathbf{k}, \mathbf{I})$. $P$ values were determined by twotailed Student's $t$-test. Genotype IDs below $x$-axis refer to the parental line of the respective M6 family. Data presented in ( $\mathbf{j}-\mathbf{I}$ ) are based on a single greenhouse experiment. Source data underlying $(\mathbf{j}-\mathbf{I})$ are provided as a Source data file.

spike-branching, e.g., a higher level of SBS and a lower frequency of Wt spikes, as compared to com2.g mutant plants. In contrast to the single mutants, all spikes of the DM plants displayed the CBS phenotypic class (Fig. 8h). DM plants therefore outperformed either single mutant in supernumerary spikelet formation; and thus, in grain number per spike (Fig. 8i). We further measured other grain-related characters (Fig. 8j-n), showing that the DM plants had the lowest TKW; most likely due to the known tradeoff with increased grain number.

To further examine the genetic interactions between COM1 and COM2 during branching inhibition of the barley spike, we performed qRT-PCR analyses. COM2 transcript levels in immature spikes of com1.a were unchanged during the two early stages tested; however, slightly lower expression was only found during later stages of development (Figs. 80 and 9a dashed red arrow). Thus, the DM analyses imply that the two loci may act partially independently/additively during branch inhibition in barley.

Putative transcriptional regulation during barley spike development. To further examine the molecular basis of COM1 branch inhibition within the barley spike, we performed qRTPCR to locate COM1 relative to other previously known spike 
a
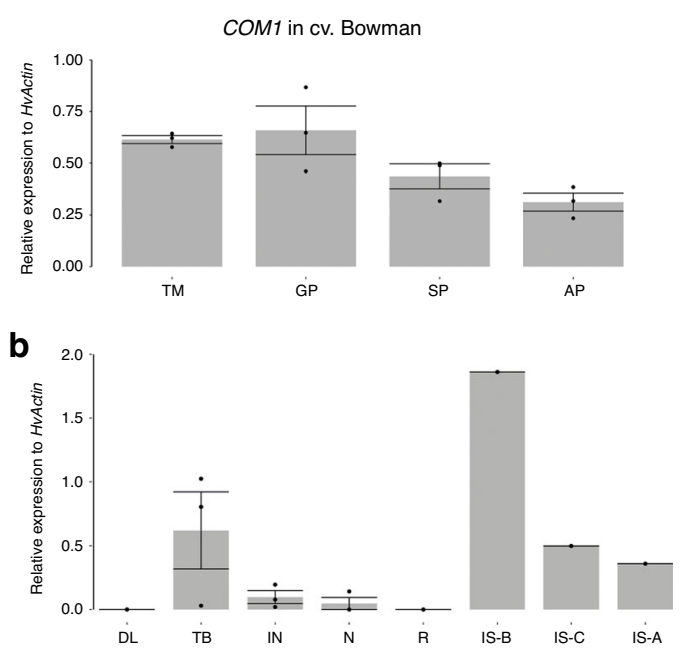

C

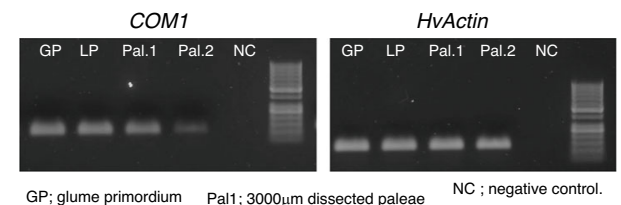

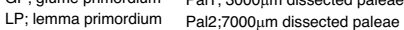

d

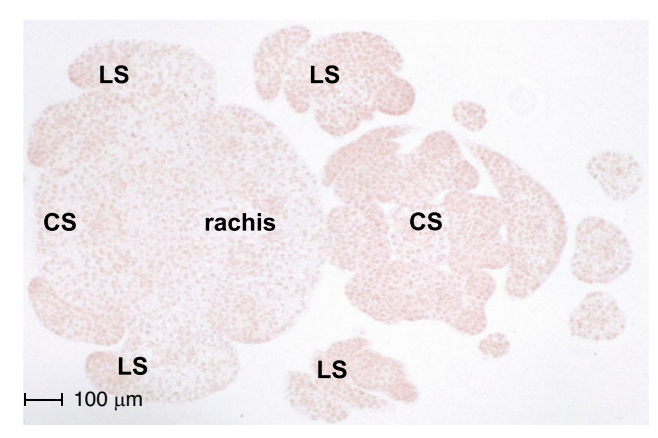

$\mathbf{e}$

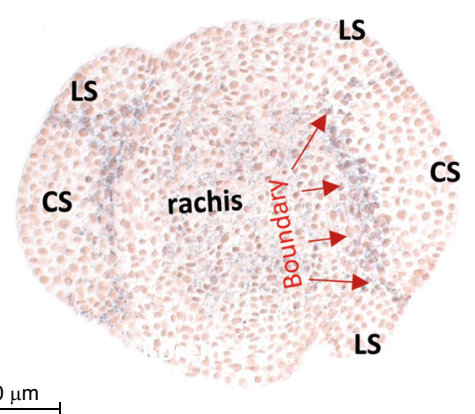

$100 \mu \mathrm{m}$

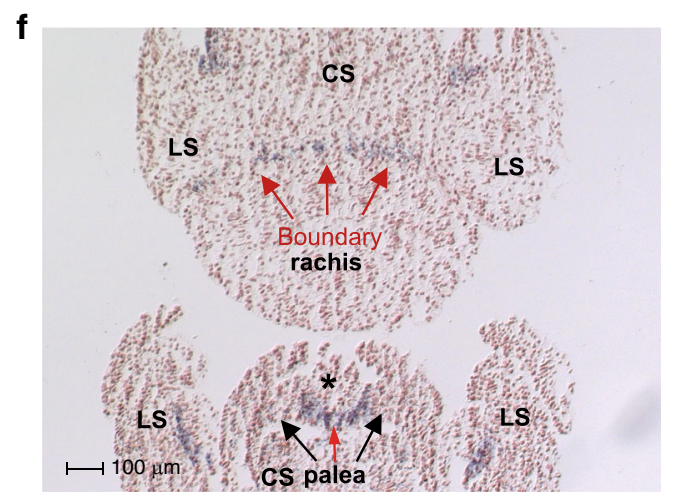

g

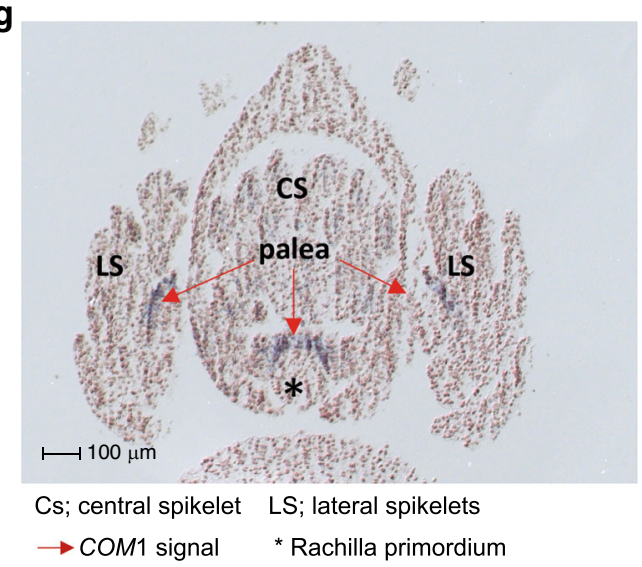

Fig. 7 Transcript analyses of COM1 in two-rowed barley. a Relative COM1 expression at different stages of immature barley spike, including TM, GP, SP, and awn primordium (AP, a stage following stamen primordium ${ }^{18}$ ) in cv. Bowman. Data are generated from three biological replicates from $15-25$ plants; mean \pm SE are reported. b Relative COM1 expression in different organs (DL; developing leaf, TB; tiller buds, IN; culm internode, N; culm node, R; Root) along with spike sections (IS-B; immature spike basal nodes, IS-C; immature spike central nodes, IS-A; immature spike apical nodes) at AP stage in cV. Bowman. Despite expression in tiller buds, no differences in tiller number was observed (Supplementary Fig. 4). Dev leaf and IM stands for developing leaf and inflorescence meristem, respectively. Data are generated from three biological replicates from 15-25 plants; mean \pm SE are reported. Note that only one biologically independent experiment was performed for IS (a-c). c Semi-qPCR of COM1 (left) and HvActin (right) mRNAs in two different stages of immature spike development, GP and LP (as positive controls), as well as in two palea samples. 50 bp Plus DNA ladder was used. d COM1 mRNA in situ control hybridization using pooled sense probes. e-g mRNA in situ hybridization of COM1 using pooled anti-sense probes. Tissues represent cross-section through a spikelet triplet at TM (e) and AP stages ( $\mathbf{f}-\mathbf{g}$ ) of barley cv. Bonus (a two-rowed Wt). Source data underlying (c, d-g) are provided as a Source data file.

architecture genes (Fig. 9a, black arrows). In addition to the interactions with COM2 (Fig. 8o), we localized COM1 downstream of VRS4 (HvRA2; orthologous to maize RAMOSA2), the main regulator of row type and branch inhibition (Supplementary Fig. $8 \mathrm{~A}-\mathrm{D})^{7,13}$ using qRT-PCR expression analyses of COM1 in the BW-NIL(vrs4.k) mutant (Supplementary Fig. 8E and Fig. 9a).

We performed comparative RNA-seq using mRNAs from immature spikes of BW and com1.a as well as the mutant progenitor, $c v$. Foma, when spike patterning begins to differ between genotypes, plus two subsequent stages (Figs. 1, 9n; see "Methods"). Differentially expressed (DE) genes were identified in comparisons of com1.a versus BW and mutant versus $c v$. Foma. We found 83 genes (Log2 FoldChanges; LFC $\mid \geq 0.5$; adjusted $P<0.05) \mathrm{DE}$ in at least one stage in both comparisons (Fig. 9b and Supplementary Figs. 9, 10): 18 and 65 genes up- and downregulated in BW-NIL(com1.a), respectively.

Among significantly downregulated genes across all three stages (Fig. 9b), we detected one SQUAMOSA PROMOTERBINDING-LIKE 8 gene (SPL8, HORVU2Hr1G111620) homologous to the boundary gene LIGULELESS 1 in maize (LG1; ZmSPL4), rice OsLG1 (OsSPL8) and hexaploid wheat TaLG1 $(T a S P L 8)^{30}$. Similar to the known maize module $(R A 2 \rightarrow$ 

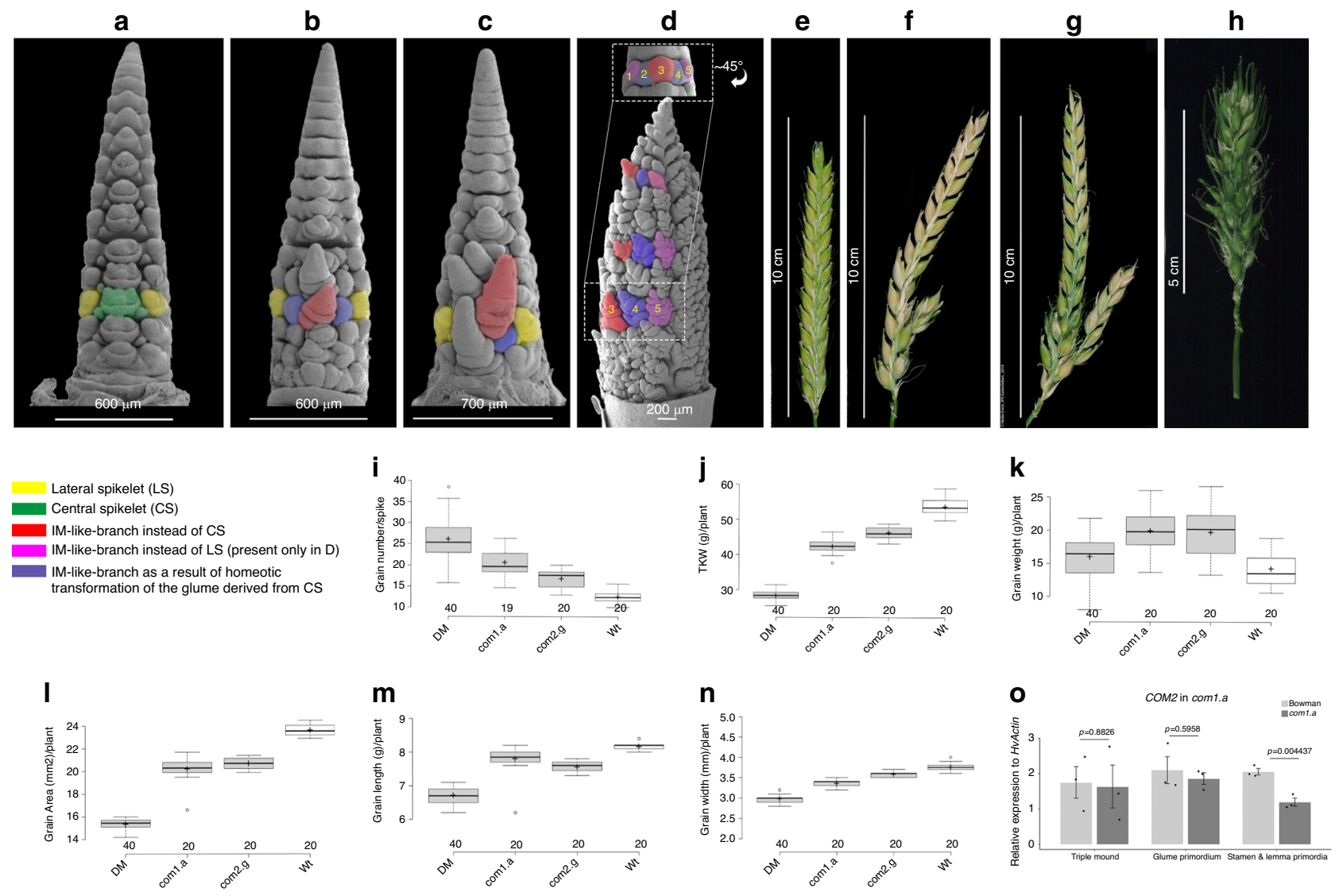

Fig. 8 Phenotypic analysis of double versus single mutants and COM2 transcript levels in com1.a. a SEM-based view of an immature spike of Wt $c V$. Bowman, single mutant com1.a (b), single mutant com2.g (c) and those of a DM mutant (d). In $\mathbf{d}$ upper panel shows basal nodes of a DM spike at GP stage with elongated CSM (as compared to Wt in Fig. $1 \mathrm{~g}$ ) and unusually enlarged glume primordia (in purple). Numbers 1-5 denote five IM-like branch meristems of a DM spike that eventually represents a putative ten-rowed spike. The lower panel is rotated by $\sim 45^{\circ}$ to the left while imaging. Please note IM-like branches 1 and 2 are invisible in the lower panel. Immature spikes (except $\mathbf{d}$ upper panel) are at similar developmental stages of early/advanced stamen primordium. Immature spikes in $(\mathbf{b}, \mathbf{c})$ represent typical SBS while $\mathbf{d}$ corresponds to the immature CBS spike class. e-h Showing mature spikes with Wt cv. Bowman depicted in (e), single mutant com2.g shown in (f) (reproduced from Poursarebani et al. ${ }^{13}$ with the kind permission from Genetics Society of America), single mutant com1.a shown in ( $\mathbf{g}$ ), and the mature spike in a DM plant displayed in (h). $\mathbf{f}, \mathbf{g}$ Represent a frequent phenotype of the SBS class at maturity while $\mathbf{d}$ represents a mature CBS phenotype. i-n Grain characters of the DM plants, and the corresponding single mutant com1.a and com2.g in comparison to the Wt cv. Bowman. Data are based on a single greenhouse experiment and on averages of 20 plants (390-540 spikes) per phenotypic class. Per box plot, plus signs show the means while center bold-lines show the medians; box limits indicate the 25th and 75th percentiles; whiskers extend 1.5 times the interquartile range, outliers are represented by circles. o Depicts COM2 transcript levels in the com1.a mutant compared to Wt cv. Bowman. Data are generated from three biological replicates from 15-25 plants per experiment; mean \pm SE of three biological replicates. Genotype differences were tested at a significance level of $P>0.05$ using the two-tailed paired Student's $t$-test. Source data underlying (i-n) are provided as a Source data file.

$W A B 1 / B A D 1 \rightarrow L G 1)^{14,15}$, we found that $V R S 4 / H v R A 2 \rightarrow$ $C O M 1 \rightarrow H v L G 1$ regulation appears to be maintained in barley. HvLG1 mRNA in situ hybridization signals showed colocalization with COM1 in the base of the forming SMs throughout the boundary region separating SMs from rachis (Fig. 9c, d). Transcriptome analysis of leaf tissues in a wheat liguleless1 mutant revealed TaSPL8 as a cell wall-related gene ${ }^{30}$. Notably, no spike-branching phenotype was reported for this erected-leaf liguleless mutant, most likely due to genetic redundancy.

Among other significantly downregulated genes in com1.a, we found important proteins associated with cell wall properties and integrity (Fig. 9b and Supplementary Fig. 9). These include HORVU5Hr1G006430, a leucine-rich repeat receptor kinase (LRR-RLK), and HORVU3Hr1G030260 belonging to the cytochrome P450 superfamily. LRR-RLKs and CYP450s are involved in lignin deposition to cell walls upon cellulose biosynthesis inhibition and during lignin biosynthesis per se, respectively ${ }^{31,32}$.
Other cell wall-related proteins include two genes encoding xyloglucan endotransglucosylase/hydrolase (XTH) 25 (HORVU7Hr1G098280 and HORVU7Hr1G098260) and barley Low Silicon Influx 1 (HvLSI1; HORVU6Hr1G075850) ${ }^{33}$, both downregulated in the mutant. These cell wall-related proteins may support COM1 involvement in regulation of cell wall mechanics of palea cells and the IM-to-SM boundary, and indirectly, putative signaling required for acquiring SM identity.

\section{Discussion}

Here we report that barley COM1 affects cell growth through regulation of cell wall properties specifically in palea and IM-toSM boundary cells; the latter provide identity signals to barley $\mathrm{SMs}^{34}$. Signaling to the SM to establish its identity is a key genetic switch by which barley inflorescences acquire spike architecture, not seen in non-Triticeae grasses.

COM1 is present only in grasses, with no true Arabidopsis ortholog; intriguingly, we observed functional differences of 
a

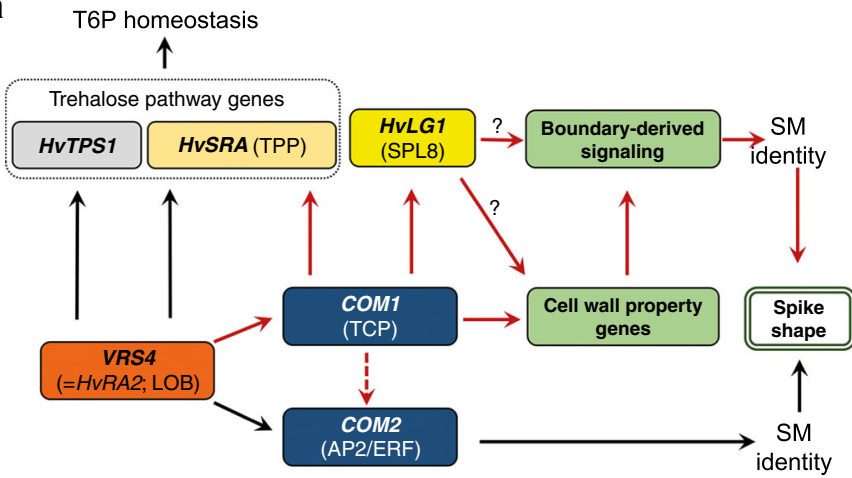

$\rightarrow$ Previously published $\rightarrow$ Identified in present study

C
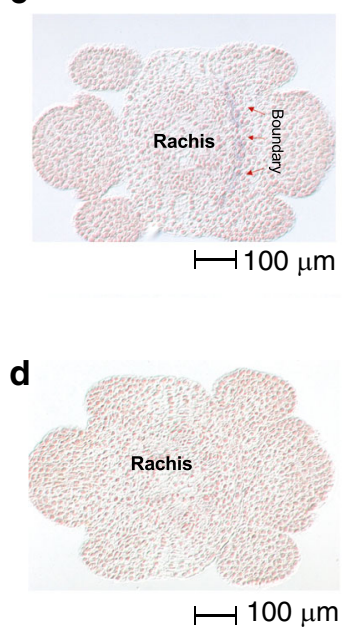

e

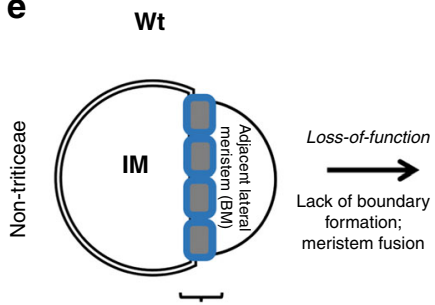

g

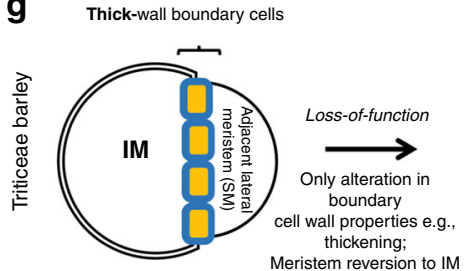

b

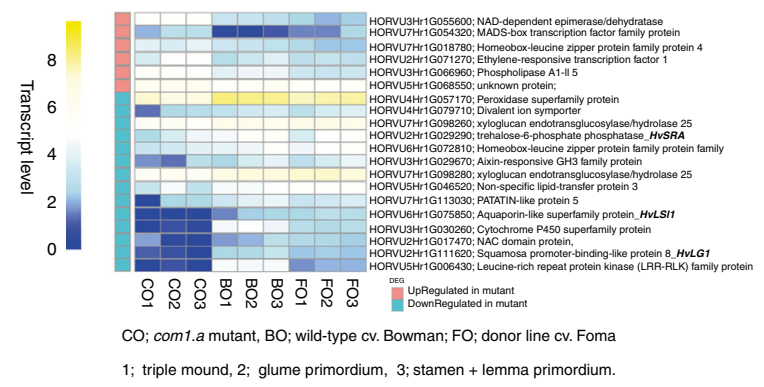

f
Putative biological function contributed by COM1

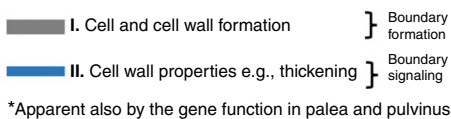

*Apparent also by the gene function in palea and pulvinus (promotes cell differentiation and proliferation, respectively). h Thin-wall boundary cells

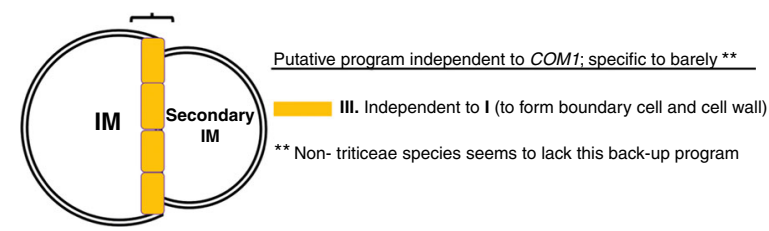

Fig. 9 COM1 transcript-based regulatory scheme, HvLG1 mRNA signals and model of COM1 functional differences from non-Triticeae. a Model of COM1 transcriptional regulation in barley deduced from either RNAseq or RT-qPCR results. Black arrows are interactions reported previously ${ }^{13,28}$ while red arrows are detected in the current study (see Supplementary Fig. 10, the legend). b RNAseq-based heat map of selected differentially expressed (DE) genes (see Supplementary Fig. 9 for the remaining DE genes). The transcript level of each gene in mutant com1.a (CO) is compared with two Wts, cv. Bowman (BO; parent of the mapping population) and cv. Foma (FO; the donor line), at three different meristematic stages. Transcript level was calculated using Eq. (1) (see "Methods"). c mRNA in situ hybridization of barley LG1 in the immature spike using the antisense (d) and sense probes (see "Methods"). Tissues represent cross-section through spikelet meristems in barley $\mathrm{cv}$. Bonus at GP stages. e-h Proposed IM-to-BM boundary formation due to Wt gene function in non-Triticeae grasses (e). Lack of boundary formation due to the loss-of-function allele (f). Of note, involvement in the alteration of the boundary cell walls within non-Triticeae species cannot be excluded. $\mathbf{g}$ Proposed IM-to-SM boundary formation in Wt barley; restriction of COM1 function to altering cell wall properties (the blue program), due to evolutionary functional differences. h Reversion to the previous identity state (IM) observed in barley com1.a due to lack of putative wall-amplified micromechanical signals needed to confer SM identity. Source data underlying (a-d) are provided as a Source data file.

COM1 between barley and non-Triticeae grass species. The differences in COM1 function was clear by comparing mutant versus wild type inflorescence phenotypes across grass species, and was further elucidated by our analysis at the cellular/molecular level. At the phenotypic level, barley COM1 inhibits spikebranching to simplify floral architecture; whereas in non-Triticeae COM1 homologs promote formation of lateral branches (e.g., up to $60 \%$ more branches in maize when compared to mutants ${ }^{15}$ ) to sustain the ancestral inflorescence complexity.

At the cellular level in non-Triticeae grasses, COM1 has evolved as a boundary formation factor, its putative ancestral role (Fig. 9e, f). Consequently, loss-of-function of COM1 homologs result in lack of boundaries and subsequent organ fusion as demonstrated by a lower number of lateral branches in maize mutants. Notably, this loss-of-function did not change the overall inflorescence architecture in non-Triticeae grasses. Barley COM1 loss-of-function, however, increases branch formation/extension, a clear deviation from the canonical spike form. As barley COM1 displayed a similar boundary mRNA expression pattern as seen in maize, we presume that barley COM1 functions through boundary signaling ${ }^{34}$, thereby affecting the identity of adjacent, destined SMs. The establishment of boundary regions in barley com 1 mutants (no organ fusion) via pathway(s) independent of COM1 (Fig. 9g, h), and thus separation of meristematic zones in this mutant, implies that barley IM-to-SM boundary cells fail to deliver proper identity-defining signals to SMs. The boundary signaling failure may perturb transcriptional programs required to establish identity to barley SMs; such meristematic cells eventually revert back to IM-like meristems forming a branch-like structure (Fig. 9h). The function of the boundary, and boundaryexpressed genes (e.g., maize RAMOSA1-3), as a signaling center for adjacent meristems, e.g. SMs, has been proposed in grasses, yet features of these signals remain unknown ${ }^{34}$. Signals associated with COM1 might include micromechanical forces derived from the formation of rigid cell walls enclosing boundary cells. Involvement of COM1 in directing such mechanical regulation is 
supported by our anatomical analysis of palea cell walls and further confirmed by our transcriptome analysis of immature barley spike samples. $H v L G 1, H v L S I$, and genes encoding one LRR-RLK, one CYP450, and two XTHs were among the most downregulated in the mutant and involved in defining cell wall properties $^{30-32,35}$. The contribution of boundary cell wall mechanics in guiding organogenesis within reproductive tissues has been well described in eudicot species ${ }^{36,37}$.

Functional differences among homologs usually include constraints on expression patterns, protein sequence/structure or participation in molecular networks, often assumed to be associated with gene duplication ${ }^{25}$. Notably, COM1 shows no sign of duplication within the barley genome and as mentioned above displays a similar expression pattern to maize ${ }^{14,15}$. Thus, COM1's functional difference and implication in boundary-derived signaling seem to be associated with its protein sequence (Fig. $2 \mathrm{~d}$ ) and the respective downstream molecular networks. Furthermore, COM1's role in regulating floral complexity-levels in grasses fits well with the view that TCP transcription factors are growth regulators and evolutionary architects of plant forms that create diversity ${ }^{38}$. They influence the final architecture of plants in response to endogenous and/or external conditions. Thus, the barley floral reductionism (from compound spike to spike shape; Fig. 1a-d) contributed by COM1, might be a response to yet unknown ecological factor(s) attributed to the origin of subfamily Triticeae.

In summary, our findings enabled the identification of a barley SM identity pathway, VRS4 (HvRA2) $\rightarrow C O M 1 \rightarrow H v L G 1$, that works partially independent of COM2 and inhibits spikebranching via boundary-defined signals (Fig. 9a and Supplementary Fig. 10). Our model of branch-inhibition in barley spikes opens a new window into grass inflorescence evolution and molecular crop breeding, and the elevated grain number per spike in com1.a/com2.g double mutants supports this notion.

\section{Methods}

Plant material. The Nordic Genetic Resource Center, the National Small Grains Collection (US Department of Agriculture), and the IPK gene bank were inquired to access 'compositum-barley' mutants (Supplementary Data 3). Bowman NIL carrying the com1.a allele ((i.e., BW-NIL(com1.a); syn. BW189 or CIho 11333)), its two-rowed progenitor Foma and Wt barley cv. Bowman were used for phenotypic descriptions, whole-genome shotgun sequencing (WGS) (see below, "Marker development") as well as SEM analysis. Plant material used to generate mapping populations is reported in the corresponding section for genetic mapping. For haplotype analysis, a core collection including of 146 diverse barley landraces and 90 diverse wild barleys were sequenced ${ }^{28,29}$.

Plant phenotyping in barley. For phenotyping, the mapping population, BW-NIL (com1.a), Bowman and the corresponding segregating populations $\left(\mathrm{F}_{2}\right.$ and $\left.\mathrm{F}_{3}\right)$ were grown side-by-side under greenhouse conditions at the IPK. For a plant to be assigned as a branched spike mutant, spike shape in all tillers was visually inspected for presence of at least one extra spikelet at any rachis node. Grain-related characters, such as weight, number, etc. were also measured at harvest for the two parental lines of the mapping population. In case of phenotyping of the barley TILLING population (see below-TILLING analysis in barley and Supplementary Data 3), other induced mutants (Supplementary Data 3 ) as well as the BW-NIL (com1.a) / BW-NIL(com2.g) double mutants (see below, "Double-mutant analysis"), visual phenotyping for variation in palea structure was also applied in addition to the aforementioned phenotyping approach used for spike-branching in $\mathrm{F}_{2}$ and $\mathrm{F}_{3}$ progenies. In case of TILLING, from the six mutants for which the spikebranching phenotype was observed at M4, only three (carrying mutation inside the protein domain; M4.15104, M4.4406, and M4. 2598) were subjected for further study in M5 generation. Here, one M4 plant was selected from which 16 M5 plants were grown and phenotyped.

Plant phenotyping in Brachypodium distachyon. An already published TILLING population and the corresponding $\mathrm{Wt}$ accession $\mathrm{Bd} 21-3$ were used for phenotyping ${ }^{39}$. It included measurement of branch angle, as proxy for pulvinus size, spikelet number per spike, floret number per spikelet and palea structure. Hence, per M4 plant, only homozygous M5 plants either with mutant genotype aa (3-4 plants) or wild type bb (3-4 plants) were selected. Per M5 plant, 10 M6 plants were grown under greenhouse conditions at the IPK and used for measurement. Thus, 30 to 40 plants per group and for each plant angles of basal spikelets in main tillers were considered for measurement. To this end, spikes were firstly imaged and then imported to the ImageJ tool (https://imagej.nih.gov/ij/index.html) for angle measurement. In case of the original wild type $\mathrm{Bd} 21-3$, five plants were grown and measured. The same set of mutant and wild type plants with their corresponding spike images were used to calculate number of spikelets per spike and number of florets per spikelet. In case of palea phenotyping: paleae were visually inspected across all spikes per plant. We detected plants with paleae being sensitive to exogenous finger-pressure; and thus, such plants were scored as mutants. A gentle finger-pressure led the mutant paleae to crash from the middle longitudinal-line so that a scissors-like structure was formed (Fig. 6a). The crashing was not evident in Wt plants even with severe exogenous finger and full hand-pressure.

Plant phenotyping in sorghum. An already published TILLING population and the corresponding $\mathrm{Wt}$ accession BTx623 were used for phenotyping ${ }^{40}$. To measure primary branch number and angle, five to eight plants per family (either M5 or M6 generation; including a Wt sorghum family cv. BTx623) were grown under greenhouse conditions at the IPK. Average primary branch (p. branch) number per panicle, e.g., per plant, was calculated by counting all p. branches that originated per rachis node for the first ten nodes (Supplementary Table 3). The node refers to the rachis area, where whorls of branches emerge. The average p. branch number per family was then used to compare with the same value obtained from Wt family BTx623. To measure the branch angle, for each plant three to four basal nodes per panicle were separately photographed. Each node contained at least one and up to five lateral branches. To cover angles of each individual branch per node, each node was photographed multiple time. Images were then imported to ImageJ for angle measurement as described for Brachypodium (see above, "Plant phenotyping in Brachypodium distachyon"). Spikelet organs, including paleae and glumes, as well as overall grain set were also visually inspected for any visible alteration.

Marker development. BW-NIL(com1.a) and two-rowed progenitor of com1.a, cv Foma, were survey-sequenced using WGS approach (see below, "Sequence information and analysis"). To detect polymorphisms, sequence information was compared against already available WGS of Bowmann ${ }^{41}$, as present in Supplementary Fig. 1. WGS paired-end reads were mapped to the Bowman reference assembly using BWA (version: 0.5 .9$)^{42}$ with a mapping quality threshold of 20 . Duplicated reads were removed using samtools (version 0.1 .18 ) $^{43}$. To detect genomic polymorphism between the two genotypes the raw set of potential variations has been called using vcftools (version 0.1.17) ${ }^{44}$. The genome alignment was visualized using Circos (version 0.52) ${ }^{45}$. Polymorphisms e.g., SNPs detected from this comparison between the two parental lines were converted to restriction enzyme-based CAPS (Cleaved Amplified Polymorphic Sequence) markers to derive a restriction based genetic marker [named as Next Generation Sequencing based markers (NGS-based markers) ${ }^{13}$. Firstly, the tool NEBcutter V2.0 (http://nc2.neb. com/NEBcutter2/) was used to detect restriction enzymes cleaving sites at the targeted SNP position; and thus, converting the SNP into a fragment-size based polymorphism (e.g., CAPS). For each targeted SNP the commercially available restriction enzyme was detected, a pair of primers spanning the restriction site was designed using the online tool BatchPrimer3 (https://wheat.pw.usda.gov/demos/ BatchPrimer3/). The PCR amplicon needed to be a single product, having a preferable length of 600-1000 bp with the SNP located at the middle. The proper primers were then used to obtain the respective PCR amplicon firstly in the parental lines of mapping population. The amplified amplicons of the parental genotypes were then subjected to restriction digestion. SNPs successfully converted into CAPS within the parental genotypes were then considered as a NGS-based genetic marker. The developed genetic markers (Supplementary Data 1) were used to screen the corresponding mapping population.

Genetic mapping and map-based cloning of com1.a. com1.a was initially proposed to be located in chromosome $5 \mathrm{HL}$ with unknown genetic position ${ }^{12}$. A barley $\mathrm{F}_{2}$ mapping population was developed by crossing Bowman NIL, i.e., BWNIL(com1.a), and barley cv. Bowman. For initial mapping 180 individuals were analyzed and genotyped using the aforementioned NGS based markers. The pattern of segregation between mutant and $\mathrm{Wt} \mathrm{F}_{2}$ plants fitted a 3:1 ratio typical for a monogenic recessive gene. Linkage analysis of segregation data was carried out using the maximum likelihood algorithm of Joinmap 4.0. Kosambi mapping function was used to convert recombination fractions into map distances. The linkage mapping was further followed by a high-resolution genetic mapping in which almost 6,000 gametes were screened with the flanking markers NGS045 and NGS049. For narrowing down the com1.a genetic interval the identified recombinants (a set of 109) were used. From 109, a set $15 \mathrm{~F}_{2}$ were labeled (Supplementary Table 1 and Supplementary Data 2) to be critical recombinants for precisely defining the com1.a genetic interval. From each of the 15 critical plants, $16 \mathrm{~F}_{3}$ progenies were evaluated for their phenotypes and marker genotypes at the com1.a candidate gene. (Supplementary Table 1 and Supplementary Data 2). Based on $F_{2}$ high-resolution mapping and $\mathrm{F}_{3}$ genetic analysis described, two tightly linked markers, NGS084 and NGS094, were taken to harvest the available barley genome sequence data (see below, "Sequence information and analysis"). A single BAC 
contig spanning $\sim 1.4 \mathrm{Mb}$ of the minimal tiling path (MTP) was identified. Genes in this region were utilized for marker development and further genetic mapping that resulted in the identification of a $\sim 380 \mathrm{~kb}$ region deleted in the mutant BW-NIL (com1.a). The deleted fragment contains a single gene (HORVU5Hr1G061270), i.e., com1.a.

Allelism test of com1 mutants. Mut.3906 mutant (Supplementary Data 3) was crossed with BW-NIL(com1.a) to test for allelism. The resultant $\mathrm{F}_{1}$ plants showed a mutant spike phenotype confirming to be allelic with com1. All alleles showed phenotypic similarities with com 1 and mutations in the COM1 gene sequences.

Double-mutant analysis. Double mutants (DM) were generated by crossing mutant BW-NIL(com1.a) to BW-NIL(com2.g), followed by selfing of the $\mathrm{F}_{1}$ progeny. All obtained $183 \mathrm{~F}_{2}$ plants were subsequently genotyped (Supplementary Data 1). In case of com2.g mutation detection, a primer pair (Com2-Bw_SfiI_FR; Supplementary Data 1) spanning the A300C haplotype (that differentiates the $\mathrm{Wt}$ Bowman allele A from com2.g mutant $\mathrm{C}$ allele at position $300 \mathrm{bp}^{13}$ ) were used for sequencing and to classify $\mathrm{F}_{2}$ genotypes for the com 2 locus. Thus, genotypic classes include $\mathrm{C} 300 \mathrm{C}$ allele as homozygous mutant, $\mathrm{AA}$ as $\mathrm{Wt}$ and CA as heterozygous. In case of coml.a, a presence/absence marker was used (Supplementary Data 1), where absence of the COM1 gene was considered as homozygous com 1.a mutant. A total number of five plants were recovered as homozygous double mutants (from $183 \mathrm{~F}_{2}$ plants) that were used for generating $\mathrm{F}_{3}$ plants used in subsequent $\mathrm{DM}$ phenotypic analysis (Fig. 8). Two DM $\mathrm{F}_{3}$ families, each consisting of 20 plants along with 20 plants from each of the single mutants and 20 wild type cv. Bowman plants, were grown and used for phenotyping (Fig. 8).

TILLING analysis in barley. For identifying further mutant alleles of COM1 in barley TILLING populations, an EMS (Ethyl methanesulfonate) treated population of $\mathrm{cv}$. Barke consisting of 10,279 individuals, for which a multidimensional pool of individual DNA samples is available ${ }^{46}$, were screened. A primer combination (Supplementary Data 1) was used to amplify the coding region of the COM1 gene. The amplicon was subjected to standard procedures using the AdvanCE ${ }^{\mathrm{TM}} \mathrm{TIL}-$ LING kit (advancedanalytical, IA, USA). Amplified products were digested with dsDNA cleavage kit followed by analysis via mutation discovery kit and gel-dsDNA reagent kit. Briefly, a premix was made containing a mismatch detecting CEL1 enzyme, namely dsDNA Cleavage Enzyme/T-Digest Buffer mixed in ratio 1:125. For digestion, two $\mu \mathrm{L}$ of heteroduplexes was used with two $\mu \mathrm{L}$ of the premix at $45^{\circ}$ for $30 \mathrm{~min}$. AdvanCETM FS96 capillary electrophoresis system was used to separate the cleaved products according to manufacturer's guidelines (Advanced Analytical, IA, USA). To distinguish the real putative mutation from the background products, sizes of two digested products after summing up were expected to give the size of the initial PCR product and only such instances were accepted. Otherwise, the original DNA pool was not considered as containing the putative mutant. All the possible mutations found using the capillary electrophoresis system were further validated by re-sequencing using Sanger sequencing and primers listed in Supplementary Data 1.

TILLING analysis in Brachypodium distachyon. Mutation screenings in the TILLING collection of chemically induced (EMS) Brachypodium mutants were performed according to Dalmais et al. ${ }^{39}$. Briefly, the method includes TILLING by NGS that consists to sequence 500 bp PCR fragments libraries prepared from 2,600 individual genomic DNA pooled in two dimensions. A dual indexing system, one placed on the 5'adaptater, and the second one on the $3^{\prime}$ adaptater, added by a twostep PCR (for primer sequence, see Supplementary Data 1) allow a direct identification of the sequence identities. The first PCR amplification is a standard PCR with target-specific primers carrying Illumina's tail (Supplementary Data 1) and 10 ng of Brachypodium genomic DNA. Two microliters of the first PCR product served as a template for the second PCR amplification, with a combination of Illumina indexed primers (Supplementary Data 1). The sequencing step of PCR fragments was done on an Illumina Miseq personal sequencer using the MiSeq Reagent Kit v3 (Illumina ${ }^{\circ}$ ) followed by quality control processes for libraries using the PippinHT system from SAGE Sciences for libraries purification, and the Bioanalyzer ${ }^{\mathrm{mt}}$ system from Agilent ${ }^{\oplus}$. To identify induced mutations, a bioinformatic pipeline called Sentinel (https://www.app.asso.fr/; under ID: IDDN.

FR.001.240004.000.R.P.2016.000.10000) was used to analyze the data sequences. Prediction of the impact of each mutation (Supplementary Data 3) was made with SIFT 5.2.2. software (Sorting Intolerant from Tolerant, https://sift.bii.a-star.edu.sg/) according to the manual guideline. The amplified ORF was also re-sequenced by Sanger sequencing using primers listed in Supplementary Data 1.

TILLING analysis in sorghum. A pedigreed sorghum mutant library was established in the inbred line BTx623, which was used to produce the sorghum reference genome. This mutant library consists of $6400 \mathrm{M} 4$ grain pools derived from EMStreated sorghum grains by single seed descent. Whole-genome sequencing of a set of 256 lines uncovered 1.8 million canonical EMS-induced mutations ${ }^{39}$. We searched the sorghum ortholog of the barley COM1 in the aforementioned sequence database to identity plants carrying mutation. To confirm the mutations, the amplified ORF was also re-sequenced by Sanger sequencing using primers listed in Supplementary Data 1.

Haplotype and network analysis. Genomic DNA from a core collection including 146 landrace and intermedium-spike barley accessions as well as 90 wild barleys was PCR-amplified using specific primers to amplify full coding sequence of the barley COM1 gene. Amplified fragments were used for direct PCR-sequencing (Sanger method; BigDye Terminator v3.1 cycle sequencing kit; Applied Biosystems). A capillary-based ABI3730xl sequencing system (Applied Biosystems) at the sequencing facility of IPK was used to separate the fluorescently terminated extension products. Sequence assembly was performed using Sequencher 5.2.2.3. Visual inspection of sequence chromatograms was carried out using Sequencher to detect the corresponding SNPs. Network analysis of the nucleotide haplotypes was carried out using TCS v1.21 software (http://darwin.uvigo.es/software/tcs.html) ${ }^{47}$.

Extraction of mRNA. For the RNA-seq study, immature spike tissues were collected from BW-NIL(com1.a) and Wt progenitor Bowman and the donor cv. Foma. Plants were grown under phytochamber conditions of $12 \mathrm{~h}$ light $\left(12^{\circ} \mathrm{C}\right)$ and $12 \mathrm{~h}$ dark $\left(8^{\circ} \mathrm{C}\right)$. Tissues were always collected at the same time slot (14:00 to 17:00) during the day at three different developmental stages, including TM and $\mathrm{GP}$, and pooled stages of LP $+\mathrm{SP}$. Three biological replicated were applied that resulted in 27 individual tissue samples. The TRIzol method (Invitrogen) was applied to extract total RNA from immature spike tissues followed by removal of genomic DNA contamination using RNAse-free DNAse (Invitrogen). RNA integrity and quantities were analyzed via Agilent 2100 Bioanalyzer (Agilent Technologies) and Qubit (Invitrogen), respectively.

Preparation and sequencing of mRNA-Seq libraries. SENSE mRNA-Seq libraries $(27=3$ repsx3 stagesx 3 genotype $)$ were prepared from $2 \mu \mathrm{g}$ total RNA according to the protocol provided by the manufacturer (Lexogen $\mathrm{GmbH}$, Vienna, Austria). Libraries were barcoded, then pooled in an equimolar manner. The average size ( $373 \mathrm{bp}$ ) of the pool was determined electrophoretically using the Agilent 4200 TapeStation System (Agilent Technologies, Inc., Santa Clara, CA, USA). The pool was quantified using quantitative $\mathrm{PCR}^{48}$. Sequencing (rapid run, paired-end sequencing, $2 \times 100$ cycles, on-board clustering) was performed using the Illumina HiSeq2500 device according to the manufacturer protocol (Illumina, San Diego, California, USA).

Analysis of the RNAseq data. The reads from all three biological replicates were pooled per stage and each pool was independently mapped to barley pseudomolecules $^{41}$, (160404_barley_pseudomolecules_masked.fasta) using TopHAT2 ${ }^{49}$. Gene expression was estimated as read counts for each gene locus with the help of featureCounts ${ }^{50}$ using the gene annotation file Hv_IBSC_PGSB_r1_HighConf.gtf and fragment per million (FPM) values were extracted from the BWA-aligned reads using Salmon ${ }^{51}$. Genes that showed FPM of 0 across all 45 samples were excluded from expression level calculations. Expression levels were normalized by TMM method. Transcript level was then calculated using the following Eq. (1):

$$
\text { Transcript level }=\log (X+1)
$$

where $X$ is the normalized expression value of a given gene. This was used to avoid problems with genes showing no expression. The $P$-values were calculated by an exact negative binomial test along with the gene-specific variations estimated by empirical Bayes method in edge $\mathrm{R}^{52}$. The Benjamini-Hochberg method was applied on the $P$-values to calculate $Q$-values and to control the false discovery rate (FDR). Differentially expressed genes (DEGs) were defined as $Q$-value $<0.05, \log 2$ fold change $>1$ or $<-1$.

Quantitative RT-PCR. Tissue sampling, RNA extraction, qualification, and quantification was performed as described above. Reverse transcription and cDNA synthesis were carried out using SuperScript III Reverse Transcriptase kit (Invitrogen). Real-time PCR was performed using QuantiTect SYBR green PCR kit (Qiagen) and the ABI prism 7900HT sequence detection system (Applied Biosystems). Each qRT-PCR comprised at least four technical replicates, and each sample was represented by three biological replicates. The Actin gene-based primers (Supplementary Data 1) were used as the reference sequence. qRT-PCR results were analyzed using SDS2.2 tool (Applied Biosystems) in which the presence of a unique PCR product was verified by dissociation analysis. Significance values were calculated using Student's $t$-test (two-tailed). The relevant primer sequences per species are detailed in Supplementary Data 1.

Phylogenetic analysis. A comprehensive analysis of TCP proteins in grasses was already available 22,23 . We, therefore, focused only on constructing a detailed phylogeny of the COM1 protein among grasses and the barley TCP genes. Thus, barley COM1 was then queried against the Ensembl Plants database (https://plants. ensembl.org/index.html) with default parameters to retrieve its orthologs or homologs from other grasses. The same database was also used to extract all barley TCP proteins. In case of COM1, protein and DNA sequence of the paralog and homologous genes from each of the grass species were retrieved. To re-check their 
homology with barley COM1, the retrieved sequences were blasted back against the barley genome. For phylogenetic analysis, protein sequences were initially aligned using the algorithm implemented in CLC sequence viewer V7.8.1 (https://www. qiagenbioinformatics.com). UPGMA tree construction method and the distance measure of Jukes-Cantor were implemented for constructing the phylogenetic tree using CLC sequence viewer. The bootstrap consensus tree inferred from 1000 replicates was taken to represent the evolutionary relationship of the sequences analyzed.

mRNA in situ hybridization. In case of COM1, three separated segments (excluding the TCP domain) each containing 300-360 bp were synthesized (probe 1 and 2, GenScript Biotech, Netherlands) or amplified (probe 3) using cDNAs isolated from immature spikes of cv. Bonus and specific primers (Supplementary Data 1). The resulting products were cloned into pBluescript II KS (+) vector (Stratagene, La Jolla, CA, USA and GenScript Biotech, Netherlands). Linearized clones by HindIII or NotI were used as templates to generate antisense (HindIII) and sense (NotI) probes using T3 or T7 RNA polymerase. In situ hybridization was conducted with a single pool of the three aforementioned probes. The stepwise method followed is illustrated comprehensively in the S1 Methods of Komatsuda et al. ${ }^{53}$. In short, tissues were fixed, dehydrated through a graded ethanol series, then series of $t$-butanol treatment, and finally embedded in Paraplast Plus (Kendall, Mansfield, MA). Slide glasses containing microtome sections ( $8-10 \mu \mathrm{m}$ thick) of tissues were deparaffinized in xylene, rehydrated through a graded ethanol series, and then air-dried. After treatment with proteinase $\mathrm{K}[(5 \mu \mathrm{g} / \mathrm{ml})$ in $100 \mathrm{mM}$ Tris. $\mathrm{HCl}, \mathrm{pH} 7.5 / 50 \mathrm{mM}$ EDTA at $37^{\circ} \mathrm{C}$ for $30 \mathrm{~min}$ and then washed twice with distilled water for $5 \mathrm{~min}$ each], slides were treated with $4 \%$ paraformaldehyde, $\mathrm{pH}$ 7.2 , for $10 \mathrm{~min}$, they were then washed twice with distilled water for $5 \mathrm{~min}$ each. Slides were subsequently treated with $0.5 \%$ acetic anhydride in $100 \mathrm{mM}$ triethanolamine, $\mathrm{pH} 8.0$, for $10 \mathrm{~min}$ at room temperature, then washed in $2^{\prime} \mathrm{SSPE}$, and dehydrated through a graded ethanol series. Hybridization was performed at $52^{\circ} \mathrm{C}$ for $>16 \mathrm{~h}$ with coverslips in hybridization buffer $(180 \mu \mathrm{L}$ per slide $)$ containing the probes. Following hybridization, successive washing steps were performed. Immunological detection of the hybridized probes was performed with a Roche digoxigenin-nucleic acid detection kit according to the manufacturer's manual. The slides were soaked with buffer 1 , and then incubated with $50 \%$ normal rabbit serum in buffer 1 containing $1 \%$ Tween 20 , for $30 \mathrm{~min}$, followed by incubation with the diluted antidigoxigenin alkaline phosphatase conjugate $(1: 1000)$ in buffer 1 containing $0.1 \%$ BSA for $1-2 \mathrm{~h}$. The slides were subsequently washed twice with buffer 1 for 10 min each, and then, rinsed with buffer 3 . After incubation at room temperature for $4-16 \mathrm{~h}$ in the dark, the colorization was stopped by immersing the slides in TE buffer $(10 \mathrm{mM}$ Tris.HCl/1 mM EDTA, $\mathrm{pH}$ 7.5). The sections were dehydrated through a graded ethanol series and then mounted in Eukitt ( $\mathrm{O}$. Kindler, Freiburg, Germany).

For the $H v L G 1$ gene, a single probe, derived from the third exon (see Fig. 9, the legend) was synthesized (GenScript Biotech, Netherlands). The aforementioned approach described for COM1 was conducted for in situ hybridization.

Scanning electron microscopy. Scanning electron microscopy (SEM) was performed on immature spike tissues at five stages including triple mound, glume, lemma, stamen, and awn primordium from greenhouse-grown plants. SEM was conducted following an established protocol in IPK-Gatersleben ${ }^{54}$. Thus, samples were fixed overnight at $4{ }^{\circ} \mathrm{C}$ in $50 \mathrm{~mm}$ cacodylate buffer ( $\left.\mathrm{pH} 7.2\right)$ containing $2 \% \mathrm{v} / \mathrm{v}$ glutaraldehyde and $2 \% \mathrm{v} / \mathrm{v}$ formaldehyde. After washing with distilled water and dehydration in an ascending ethanol series, samples were critical point-dried in a Bal-Tec critical point dryer (Leica microsystems; https://www.leica-microsystems. com). Dried specimens were attached to carbon-coated aluminum sample blocks and gold-coated in an Edwards S150B sputter coater (Edwards High Vacuum Inc.; http://www.edwardsvacuum.com). Probes were examined in a Zeiss Gemini30 scanning electron microscope (Carl Zeiss microscopy GmbH; https:// www.zeiss.de) at $10 \mathrm{kV}$ acceleration voltage. Images were digitally recorded.

DNA preparation. DNA was extracted from leaf samples at the seedling stage following an established protocol in IPK-Gatersleben. Thus, $200-400 \mathrm{mg}$ of plant material was placed in an $2 \mathrm{ml}$ Eppendorf tube. The tube containing two steel balls were then frozen in liquid nitrogen. The frozen tubes were placed in the grinding machine for $30 \mathrm{~s}$ on program 7 (P7) at the speed of 28 spins/sec. The Eppi-holder were once rotated $180^{\circ}$ during the grinding step. $800 \mu \mathrm{L}$ of the extraction buffer (0.1 M TRIS-HCl, pH 8, $10 \mathrm{mM}$ EDTA, $0.1 \mathrm{M} \mathrm{NaCl}, 1 \% \mathrm{~N}$-Lauryl-Sarcosin) was then added to each tube, followed by high speed vortex. Subsequently, $800 \mu \mathrm{L}$ of phenol/chloroform/isoamylalcohol (25:24:1) was then added, followed by a shaking at $300 \mathrm{rpm}$ for $5 \mathrm{~min}$. The Eppendorf tube was then centrifuged $(10 \mathrm{~min}, 20,853 \times$ $g$, room temperature). The resulted supernatant was transferred into a new $1.5 \mathrm{ml}$ Eppendorf tube. $60 \mu \mathrm{L} 3 \mathrm{M} \mathrm{NaAc}, \mathrm{pH} 5.2(1 / 10 \mathrm{Vol})$ and $600 \mu \mathrm{L}$ Isopropanol $\left(-20^{\circ} \mathrm{C}\right)(3 / 4 \mathrm{Vol})$ was added into each new Eppendorf tube containing the supernatant. The tubes were then gently mixed by inverting the Eppendorf tube until streaks of DNA were visible. The tubes were then centrifuged again $\left(20,853 \times g\right.$ for $10 \mathrm{~min}$ at $\left.4{ }^{\circ} \mathrm{C}\right)$. The resulted supernatant was then removed to leave the resulted DNA pellet intact. $800 \mu \mathrm{L}$ of $70 \%$ Ethanol was added and vortex to release the pellet from the bottom of the Eppendorf tube. Centrifugation was repeated (at $20,853 \times g$ for $5 \mathrm{~min}$ at $4{ }^{\circ} \mathrm{C}$ ). The supernatant was completely removed without disturbing the DNA pellet. The resulted DNA pellet was dried up at room temperature for $30 \mathrm{~min}$ (with the tube-caps opened). 50-100 $\mu \mathrm{L}$ RNase solution $(40 \mu \mathrm{g} / \mathrm{ml}$ in TE, $10: 1, \mathrm{pH} 8)$ was added and incubated $\left(1 \mathrm{~h}, 37^{\circ} \mathrm{C}\right)$. The tubes were then incubated overnight on a shaker at $4^{\circ} \mathrm{C}$. A $2 \mu \mathrm{g}$ aliquot of DNA from each sample was separated on a $0.7 \%(\mathrm{w} / \mathrm{v})$ agarose gel at $35 \mathrm{~V}$ for $20 \mathrm{~h}$ and stained with ethidium bromide. Plants for which the DNA was prepared included barley, Sorghum, and Barchypodium. The DNA was extracted either from mapping population, TILLING mutants, or both.

Palea anatomical and TEM analyses. For our anatomical studies and for transmission electron microscopy (TEM), plant material consisting of intact spikes was collected shortly before anthesis. Spikelets containing no grains were used for dissecting paleae that were subsequently stored in fixative ( $4 \%$ FA, $1 \%$ GA in $50 \mathrm{mM}$ phosphate buffer). Central spikelets (in case of barley) were isolated and placed in a $15 \mathrm{ml}$ test tube containing $10 \mathrm{ml}$ fixative, followed by extensive degassing until all probes had settled. Material was stored in a fridge until use. After three washes with A. D., lemma and palea were isolated by cutting away a small part at the base of the spikelet. Isolated paleae were placed in a flat-bottomed mold filled with $4 \%$ liquid agarose $\left(\sim 60^{\circ} \mathrm{C}\right)$. After setting, agarose blocks were removed from the mold and the encapsulated palea was cut into $1-2 \mathrm{~mm}$ wide sections using fresh razor blades. Embedding the cuttings in agarose facilitated preventing unnecessary damage to the probes. After embedding in Spurr resin semithin sections of $2 \mu \mathrm{M}$ were cut on a Leica Ultracut. Sections were allowed to be baked in a droplet of $0.02 \%$ Methylene blue/ Azur blue on a heating plate set at $90^{\circ} \mathrm{C}$. Recordings were made using a Keyence VHX-5000 digital microscope (Keyence Germany GmbH, Neu-Isenburg, Germany)

Sequence information and analysis. The sequence information for the BAC contigs 44150 spanning the interval between NGS084 and NGS094 was made available from the international barley sequencing consortium (through Nils Stein). The sequence information was used for marker development during highresolution mapping, map-based cloning, and COM1 gene identification. Later on, the initial contig 44150 sequence information was re-checked and confirmed with the high-quality barley genome assembly and annotation data ${ }^{41}$. Barley genome sequence assembly has previously been publicly available https://doi.org/10.5447/ IPK/2016/34 and http://webblast.ipk-gatersleben.de/barley_ibsc/41. Likewise, this sequence information of contig 44150 (as part of the barley assembly) can be obtained by performing a BLAST-N search against the barley genome assembly (http://webblast.ipk-gatersleben.de/barley_ibsc/) using primer sequences of genetic markers NGS084 and NGS094 (Supplementary Data 1).

Whole-genome shotgun sequencing of BW-NIL(com1.a). A whole-genome shotgun library was constructed using standard procedures (TruSeq DNA; Illumina) and quantified using real-time PCR. Cluster formation using the cBot device and paired-end sequencing (HiSeq2000, $2 \times 101$ cycles) were performed according to the manufacturer's instructions (Illumina).

Statistics and reproducibility. In case of representative experiments (such as micrographs), an initial visual inspection of the respective Wt or mutant phenotype (e.g., spike-branching, palea phenotype or the pulvinus morphology in sorghum and Brachypodium) was performed on at least $20-25$ plants per genotype. This inspection and the respective observation were confirmed and reproduced by conventional light-microscopy (for instance by checking for pulvinus presence/ morphology) using at least 10 different plants per species per $\mathrm{Wt}$ and mutant class. Such representative experiments were then followed by using extremely sensitive tools, e.g., SEM and TEM, in which each experiment was repeated multiple times (at least 3 times) on $\mathrm{Wt}$ and mutant randomly selected samples.

In case of transcript analyses, such as RNAseq and qRT-PCR, each experiment was performed at least in three independent replicates (except otherwise mentioned in the legend); each containing 15-25 plants per genotype. This number of plants was required to obtain enough meristematic tissues required for extraction of mRNA. Plants per genotypes were selected randomly from larger groups of plants grown in uniform growth conditions (see above- extraction of mRNA). For mRNA in situ hybridization, the analysis per gene was performed on three different experiments, each at least three different meristematic stages. For significance test, see the corresponding section or figure legends (Figs. 1, 5-8, and the supplementary Figs. 2 and 4$)$.

Reporting summary. Further information on research design is available in the Nature Research Reporting Summary linked to this article.

\section{Data availability}

Data supporting the findings of this work are available within the paper and its Supplementary Information files. A Reporting summary for this Article is available as a Supplementary Information file. The datasets generated and analyzed during the current study are available from the corresponding authors upon request. For requesting barley mutants, material transfer agreement (MTA) with IPK Gatersleben is needed. The RNAseq data, the whole-genome shotgun (WGS) sequences of com1.a mutant, as well as the 
COM1 sequence have been submitted to the European Nucleotide Archive under accession PRJEB35746, PRJEB35761, and PRJEB36813, respectively. COM1 sequence is also available in the Ensembl Plants database with gene ID HORVU5Hr1G061270. Source data are provided with this paper.

Received: 1 February 2020; Accepted: 15 September 2020; Published online: 12 October 2020

\section{References}

1. Zhang, D. B. \& Yuan, Z. Molecular control of grass inflorescence development. Annu. Rev. Plant Biol. 65 65, 553-578 (2014).

2. Vegetti, A. \& Anton, A. M. Some evolution trends in the inflorescence of Poaceae. Flora 190, 225-228 (1995).

3. Kellogg, E. A. et al. Early inflorescence development in the grasses (Poaceae). Front. Plant Sci. 4, 250 (2013).

4. Endress, P. K. Disentangling confusions in inflorescence morphology: patterns and diversity of reproductive shoot ramification in angiosperms. J. Syst. Evolution 48, 225-239 (2010).

5. Remizowa, M. V., Rudall, P. J., Choob, V. V. \& Sokoloff, D. D. Racemose inflorescences of monocots: structural and morphogenetic interaction at the flower/inflorescence level. Ann. Bot. 112, 1553-1566 (2013).

6. Kellogg, E. A. Evolutionary history of the grasses. Plant Physiol. 125, 1198-1205 (2001).

7. Koppolu, R. \& Schnurbusch, T. Developmental pathways for shaping spike inflorescence architecture in barley and wheat. J. Integr. Plant Biol. 61, 278-295 (2019).

8. Lemmon, Z. H. et al. The evolution of inflorescence diversity in the nightshades and heterochrony during meristem maturation. Genome Res. 26, 1676-1686 (2016).

9. Malcomber, S. T., Preston, J. C., Reinheimer, R., Kossuth, J. \& Kellogg, E. A. Developmental gene evolution and the origin of grass inflorescence diversity. Adv. Botanical Res. 44 44, 425-481 (2006).

10. Vollbrecht, E., Springer, P. S., Goh, L., Buckler, E. S. \& Martienssen, R. Architecture of floral branch systems in maize and related grasses. Nature 436, 1119-1126 (2005).

11. Lundqvist, U. Scandinavian mutation research in barley - a historical review. Hereditas 151, 123-131 (2014).

12. Druka, A. et al. Genetic dissection of barley morphology and development. Plant Physiol. 155, 617-627 (2011).

13. Poursarebani, N. et al. The genetic basis of composite spike form in barley and 'miracle-wheat'. Genetics 201, 155-165 (2015).

14. Lewis, M. W. et al. Gene regulatory interactions at lateral organ boundaries in maize. Development 141, 4590-4597 (2014).

15. Bai, F., Reinheimer, R., Durantini, D., Kellogg, E. A. \& Schmidt, R. J. TCP transcription factor, BRANCH ANGLE DEFECTIVE 1 (BAD1), is required for normal tassel branch angle formation in maize. Proc. Natl Acad. Sci. USA 109, 12225-12230 (2012).

16. Yuan, Z. et al. RETARDED PALEA1 controls palea development and floral zygomorphy in rice. Plant Physiol. 149, 235-244 (2009).

17. Zeng, D.-D. et al. DBOP specifies palea development by suppressing the expansion of the margin of palea in rice. Genes Genomics 38, 1095-1103 (2016).

18. Kirby, E. J. M., Appleyard, M., National Agricultural, C. \& Arable, U. Cereal development guide, (Arable Unit, National Agricultural Centre, Kenilworth, England, 1986).

19. Cosgrove, D. Biophysical control of plant-cell growth. Annu. Rev. Plant Physiol. 37, 377-405 (1986).

20. Cosgrove, D. J. Plant cell wall extensibility: connecting plant cell growth with cell wall structure, mechanics, and the action of wall-modifying enzymes. $J$. Exp. Bot. 67, 463-476 (2016).

21. Martin-Trillo, M. \& Cubas, P. TCP genes: a family snapshot ten years later. Trends Plant Sci. 15, 31-39 (2010).

22. Zhao, J. et al. Genome-wide identification and expression profiling of the TCP family genes in spike and grain development of wheat (Triticum aestivum L.). Front. Plant Sci. 9, 1282 (2018).

23. Francis, A. et al. Comparative phylogenomic analysis provides insights into TCP gene functions in Sorghum. Sci. Rep. 6, 38488 (2016).

24. Mondragon-Palomino, M. \& Trontin, C. High time for a roll call: gene duplication and phylogenetic relationships of TCP-like genes in monocots. Ann. Bot. 107, 1533-1544 (2011).

25. Studer, R. A. \& Robinson-Rechavi, M. How confident can we be that orthologs are similar, but paralogs differ? Trends Genet. 25, 210-216 (2009).

26. Jiao, Y. P. et al. MSD1 regulates pedicellate spikelet fertility in sorghum through the jasmonic acid pathway. Nat. Commun. 9, 822 (2018).
27. Aguilar-Martinez, J. A., Poza-Carrion, C. \& Cubas, P. Arabidopsis BRANCHED1 acts as an integrator of branching signals within axillary buds. Plant Cell 19, 458-472 (2007)

28. Koppolu, R. et al. Six-rowed spike4 (Vrs4) controls spikelet determinacy and row-type in barley. Proc. Natl Acad. Sci. USA 110, 13198-13203 (2013).

29. Russell, J. et al. Exome sequencing of geographically diverse barley landraces and wild relatives gives insights into environmental adaptation. Nat. Genet. 48, 1024-+ (2016).

30. Liu, K. et al. Wheat TaSPL8 modulates leaf angle through auxin and brassinosteroid signaling. Plant Physiol. 181, 179-194 (2019).

31. Van der Does, D. et al. The Arabidopsis leucine-rich repeat receptor kinase MIK2/LRR-KISS connects cell wall integrity sensing, root growth and response to abiotic and biotic stresses. PLos Genet. 13, e1006832 (2017).

32. Gou, M. Y., Ran, X. Z., Martin, D. W. \& Liu, C. J. The scaffold proteins of lignin biosynthetic cytochrome P450 enzymes. Nat. Plants 4, 299-310 (2018).

33. Chiba, Y., Mitani, N., Yamaji, N. \& Ma, J. F. HvLsi1 is a silicon influx transporter in barley. Plant J. 57, 810-818 (2009).

34. Whipple, C. J. Grass inflorescence architecture and evolution: the origin of novel signaling centers. N. Phytol. 216, 367-372 (2017).

35. Hara, Y., Yokoyama, R., Osakabe, K., Toki, S. \& Nishitani, K. Function of xyloglucan endotransglucosylase/hydrolases in rice. Ann. Bot. 114, 1309-1318 (2014).

36. Tameshige, T., Hirakawa, Y., Torii, K. U. \& Uchida, N. Cell walls as a stage for intercellular communication regulating shoot meristem development. Front. Plant Sci. 6, 324 (2015).

37. Landrein, B. \& Ingram, G. Connected through the force: mechanical signals in plant development. J. Exp. Bot. 70, 3507-3519 (2019).

38. Manassero, N. G., Viola, I. L., Welchen, E. \& Gonzalez, D. H. TCP transcription factors: architectures of plant form. Biomol. Concepts 4, 111-127 (2013).

39. Dalmais, M. et al. A TILLING Platform for Functional Genomics in Brachypodium distachyon. PLoS ONE 8, e65503 (2013).

40. Jiao, Y. P. et al. A sorghum mutant resource as an efficient platform for gene discovery in grasses. Plant Cell 28, 1551-1562 (2016).

41. Mascher, M. et al. A chromosome conformation capture ordered sequence of the barley genome. Nature 544, 427-433 (2017).

42. Li, H. \& Durbin, R. Fast and accurate short read alignment with BurrowsWheeler transform. Bioinformatics 25, 1754-1760 (2009).

43. $\mathrm{Li}, \mathrm{H}$. et al. The sequence alignment/map format and SAMtools. Bioinformatics 25, 2078-2079 (2009).

44. Danecek, P. et al. The variant call format and VCFtools. Bioinformatics 27, 2156-2158 (2011).

45. Krzywinski, M. et al. Circos: an information aesthetic for comparative genomics. Genome Res. 19, 1639-1645 (2009).

46. Gottwald, S., Bauer, P., Komatsuda, T., Lundqvist, U. \& Stein, N. TILLING in the two-rowed barley cultivar 'Barke' reveals preferred sites of functional diversity in the gene HvHox1. BMC Res Notes 2, 258 (2009).

47. Clement, M., Posada, D. \& Crandall, K. A. TCS: a computer program to estimate gene genealogies. Mol. Ecol. 9, 1657-1659 (2000).

48. Mascher, M. et al. Barley whole exome capture: a tool for genomic research in the genus Hordeumand beyond. Plant J. 76, 494-505 (2013).

49. Kim, D. et al. TopHat2: accurate alignment of transcriptomes in the presence of insertions, deletions and gene fusions. Genome Biol. 14, R36 (2013).

50. Liao, Y., Smyth, G. K. \& Shi, W. featureCounts: an efficient general purpose program for assigning sequence reads to genomic features. Bioinformatics $\mathbf{3 0}$, 923-930 (2014)

51. Patro, R., Duggal, G., Love, M. I., Irizarry, R. A. \& Kingsford, C. Salmon provides fast and bias-aware quantification of transcript expression. Nat. Methods 14, 417-419 (2017).

52. Robinson, M. D., McCarthy, D. J. \& Smyth, G. K. edgeR: a Bioconductor package for differential expression analysis of digital gene expression data. Bioinformatics 26, 139-140 (2010).

53. Komatsuda, T. et al. Six-rowed barley originated from a mutation in a homeodomain-leucine zipper I-class homeobox gene. Proc. Natl Acad. Sci. USA 104, 1424-1429 (2007)

54. Lolas, I. B. et al. The transcript elongation factor FACT affects Arabidopsis vegetative and reproductive development and genetically interacts with HUB1/2. Plant J. 61, 686-697 (2010).

\section{Acknowledgements}

We thank the IPK Genebank, Germany, the Nordic Genetic Resources Center (NordGen), Alnarp, Sweden and the US Department of Agriculture-Agricultural Research Service (USDA-ARS), the National Small Grains Collection, Aberdeen (ID) for providing the mutants and germplasm for haplotype analysis. The authors would like to thank Dr. Shun Sakuma for fruitful discussions and help in conducting mRNA in situ hybridizations. We are thankful to Anne Fiebig for help with data submission to ENA, Julie-Sophie Himpe and Andreas Bähring for graphical image support and Sandra Driesslein, Jenny 
Knibbiche, Mechthild Pürschel, Ines Walde, Kerstin Wolf, Marion Benecke, and Kirsten Hoffie for excellent technical assistance. During this study, research in the Schnurbusch laboratory received financial support from the Federal Ministry of Education and Research (BMBF) FKZ 0315954A and 031B0201A, HEISENBERG Program of the German Research Foundation (DFG), grant No. SCHN 768/8-1 and SCHN 768/15-1, as well as IPK core budget.

\section{Author contributions}

T.S. conceived the idea for the study, designed and monitored experiments, and analyzed data. N.P. expanded the idea for the study, designed and performed experiments and analyzed data; C.T. executed the mRNA in situ hybridizations. M.M. conducted microscopic analyses of cellular structures in paleae. T.N. analyzed RNA-seq data. U.L. provided irregular and intermedium barley spike mutants. T.R. executed SEM analyses. T.Schm. conducted sequence read mapping to published barley genomic sequences for SNP calling. R.B., A.H., and L.A. performed the initial whole-genome shotgun sequencing of the parental genotypes for mapping. R.K. was involved in the phenotypic analysis of com1. $a$ and RT-qPCR analyses of COM1 in barley vrs 4 mutant. H.M.Y. provided sequences from a barley diversity panel for haplotype analysis and was involved in the RT-qPCR analysis. R.S., M.D., and A.B. provided the Brachypodium TILLING resource; N.S. provided the barley TILLING resource; Z.X. provided the sorghum TILLING resource. N.P. and T.S. wrote the paper, including contributions from co-authors. All authors have seen and agreed upon the final version of the manuscript.

\section{Funding}

Open Access funding enabled and organized by Projekt DEAL.

\section{Competing interests}

The authors declare no competing interests.

\section{Additional information}

Supplementary information is available for this paper at https://doi.org/10.1038/s41467020-18890-y.

Correspondence and requests for materials should be addressed to N.P. or T.S.

Peer review information Nature Communications thanks Elizabeth Kellogg, Gary Muehlbauer and the other, anonymous, reviewer(s) for their contribution to the peer review of this work. Peer reviewer reports are available.

Reprints and permission information is available at http://www.nature.com/reprints

Publisher's note Springer Nature remains neutral with regard to jurisdictional claims in published maps and institutional affiliations.

\section{(c) (i)}

Open Access This article is licensed under a Creative Commons Attribution 4.0 International License, which permits use, sharing, adaptation, distribution and reproduction in any medium or format, as long as you give appropriate credit to the original author(s) and the source, provide a link to the Creative Commons license, and indicate if changes were made. The images or other third party material in this article are included in the article's Creative Commons license, unless indicated otherwise in a credit line to the material. If material is not included in the article's Creative Commons license and your intended use is not permitted by statutory regulation or exceeds the permitted use, you will need to obtain permission directly from the copyright holder. To view a copy of this license, visit http://creativecommons.org/ licenses/by/4.0/.

(C) The Author(s) 2020 